(C) Cambridge University Press 2008

\title{
Destabilization of mixed Rossby gravity waves and the formation of equatorial zonal jets
}

\author{
Bach Lien Hua ${ }^{1,{ }^{*}}$, Marc D'Orgeville ${ }^{1}$, Mark D. Fruman ${ }^{1}$, Claire Menesguen $^{1}$, Richard \\ Schopp ${ }^{1}$, Patrice Klein $^{1}$ and Hideharu Sasaki ${ }^{2}$
}

\footnotetext{
${ }^{1}$ Laboratoire de Physique des Océans, IFREMER, BP 70, 29280 Plouzané, France

${ }^{2}$ Earth Simulator Center, Yokohama, Japan
}

*: Corresponding author : Bach Lien Hua, email address : Bach.Lien.Hua@ifremer.fr

\begin{abstract}
:
The stability of mixed Rossby gravity (MRG) waves has been investigated numerically using threedimensionally consistent high-resolution simulations of the continuously stratified primitive equations. For short enough zonal wavelength, the westward phase propagating MRG wave is strongly destabilized by barotropic shear instability leading to the formation of zonal jets. The large-scale instability of the zonally short wave generates zonal jets because it consists primarily of sheared meridional motions, as shown recently for the short barotropic Rossby wave problem.

Simulations were done in a variety of domain geometries: a periodic re-entrant channel, a basin with a short MRG wave forced in its western part and a very long channel initialized with a zonally localized MRG wave. The characteristics of the zonal jets vary with the geometry. In the periodic re-entrant channel, barotropic zonal jets dominate the total flow response at the equator and its immediate vicinity. In the other cases, the destabilization leads to zonal jets with quite different characteristics, especially in the eastward group propagating part of the signal. The most striking result concerns the formation of zonal jets at the equator, alternating in sign in the vertical, with vertical scale short compared to the scale of the forcing or initial conditions.
\end{abstract}

A stability analysis of a simplified perturbation vorticity equation is formulated to explain the spatial scale selection and growth rate of the zonal jets as functions of the characteristics of the basic state MRG wave. For both types of zonal jets, the model predicts that their meridional scales are comparable to the zonal scale of the MRG wave basic state, while their growth rates scale as $\mu$ xs221D Fr |k|, where $\mathrm{Fr}$ is the Froude number of the meridional velocity component of the basic state and $\mathrm{k}$ its non-dimensional zonal wavenumber. The vertical scale of the baroclinic zonal jets corresponds to the dominant harmonic ppeak of the basic state in the fastest growing mode, given by ppeak $\approx 0.55 \mathrm{k} 2$. Thus, the shorter the zonal wavelength of the basic state MRG wave, the narrower the meridional scale of the zonal jets, both barotropic and baroclinic, with the vertical scale of the baroclinic jets being tied to their meridional scale through the equatorial radius of deformation, which decreases as the square root of the vertical wavenumber. The predictions of the spatial scales are in both qualitative and quantitative agreement with the numerical simulations, where shorter vertical scale baroclinic zonal jets are favoured by shorter 
wavelength, longer period MRG wave basic states, with the vertical mode number increasing as the square of the MRG wave period.

An appendix deals with the case of zonally long and intermediate wavelength MRG waves, where a weak instability regime causes a moderate adjustment involving resonant triad interactions without leading to jet formation. For eastward phase propagating waves, adjustment does not lead to significant angular momentum redistribution.

\section{Introduction}

The most striking feature of low-frequency zonal flow in the deep equatorial ocean is its high degree of symmetry about the equator. At the ocean depth scale, a substantial latitudinal homogenization of angular momentum appears as the background ambient state (Hua et al. 1997; Bourles et al. 2003), upon which are superposed several kinds of smaller vertical scale phenomena. At the $350-700 \mathrm{~m}$ vertical scale are the ubiquituous Equatorial Deep Jets (EDJs), east-west currents that alternate in direction with depth and are trapped in the immediate vicinity of the equator (Firing 1987; Gouriou et al. 2001). At vertical scales less than $100 \mathrm{~m}$, meridionally-extended density layering is observed throughout the water column (d'Orgeville et al. 2004). Away from the equator, extra-equatorial jets with predominantly eastward flow and of larger vertical scales reach their maxima at about $2^{\circ}$ latitude (Gouriou et al. 2001). Further away from the equator, barotropic zonal jets that alternate in sign with latitude are observed in situ and in the most recent general circulation model simulations (Richards et al. (2006), Baldwin et al. (2007) and references therein).

Previous attempts at explaining the observed large-scale angular momentum homogenization invoked inertial instability as the underlying mechanism (Hua et al. 1997). On the equatorial $\beta$-plane, inertial instability occurs whenever the maximum angular momentum $M=u-\frac{1}{2} \beta y^{2}$ (where $u$ is the zonal velocity, $y$ is latitude and $\beta$ is the meridional gradient of planetary vorticity at the equator) is displaced from the equator and is thus triggered whenever a zonal flow is not symmetric with respect to $y=0$. Among free equatorial waves, Mixed Rossby Gravity (MRG) waves represent the simplest such symmetry breaking in angular momentum between the two hemispheres, since their zonal velocity component is antisymmetric in $y$. d'Orgeville \& Hua (2005) have studied the stability of zonally symmetric MRG waves and shown that they are prone to a parametric subharmonic inertial instability (PSII) induced by the oscillating meridional shear $\frac{\partial U}{\partial y}$ of the wave (see also Natarov et al. (2008)). This instability also leads to an angular momentum homogenization via the breaking of high-baroclinic waves excited through subharmonic triadic resonances. In contrast with the ultra-violet character of the classical steady inertial instability problem where the vertical scale selection problem requires vertical diffusion or higher order nonlinear dynamics to become well posed (Dunkerton 1981; Hua et al. 1997; Griffiths 2003b), PSII has a well-defined inviscid vertical scale selection favouring high baroclinic modes through a subharmonic resonance. This PSII 
mechanism efficiently triggers a meridionally-extended density layering such as is observed in the deep equatorial ocean (d'Orgeville et al. 2004).

The present work is an extension of the destabilization study of d'Orgeville \& Hua (2005) from the zonally-symmetric to the zonally-variable MRG waves case. Our focus on the MRG wave out of all equatorial free waves stems from its mixed character, as pointed out by Matsuno (1966), " this wave connects the two families of inertio-gravity and Rossby waves, and because of its existence, the vacant space in the frequency diagram is lost ". This motivated a more systematic study of its destabilization mechanisms as potential pathways for exciting zero-frequency linear eigenmodes that are large-scale zonal flows. The MRG wave can furthermore be interpreted as a modification of an inertial oscillation or a boundary wave along an open boundary (Iga 1995) and is thus generically involved in symmetrybreaking mechanisms at the equator.

For the short westward-propagating waves limit, the dynamics of a MRG wave is known to be similar to the dynamics of a short barotropic Rossby wave with predominantly meridional motions (Matsuno 1966). For the mid-latitude $\beta$-plane, Gill (1974) has examined the stability of planetary waves and shown in particular that a short Rossby wave is prone to a longer wave instability that will favour the creation of low-frequency zonal flows if the base wave flow involves predominantly meridional motions. In the absence of the $\beta$ effect, such results are related to those of Meshalkin \& Sinai (1961) for the short wave limit of the Kolmogorov sinusoidal base flow in the two-dimensional Navier Stokes equations, with an instability occurring preferentially for perturbations (i) of larger wavelength and (ii) with wavevectors perpendicular to the wavevector of the Kolmogorov base flow, and is therefore quite anisotropic. Such a large-scale instability is deemed to be the quintessential mechanism underlying the well-known energy cascade of two-dimensional turbulence (Sivashinsky 1985). In the presence of the $\beta$-effect, an additional source of anisotropy is introduced by differential rotation that tends to align streamlines in the zonal direction along lines of constant planetary vorticity $f$ (Rhines 1994; Vallis \& Maltrud 1993). These issues have since received considerable attention (e.g Frisch et al. 1994) and a thorough discussion and complete references can be found in Manfroi \& Young (1999), Manfroi \& Young (2002) and Lee \& Smith (2002). The short Rossby wave base state corresponds to the case where the two sources of anistropy reinforce each other, involving primarily meridional motions across isolines of constant planetary vorticity. The resulting large-scale instability generates zonal jets, the nonlinear properties of which were studied by Manfroi and Young (1999) for the mid-latitude $\beta$-plane.

Since the early works of Boyd and Ripa in the 1980s, nonlinear dynamics of equatorial waves have been studied by many authors (e.g. Boyd 1998; Majda 2003; Majda \& Klein 2003; Reznik \& Zeitlin 2007), mainly in the context of shallow water dynamics of a one- or two-layer model. For the case of the MRG wave, Bouchut et al. (2005) have shown numerically that in a one-layer shallow water model eastwardpropagating MRG waves are subject to shock formation because of their unbalanced dynamics in the zonal direction, whereas westward-propagating MRG waves are stable due to their balanced dynamics. All of the above authors thus focussed upon the behaviour of low baroclinic modes. The approach used in 
the present study has been to investigate numerically the destabilization of short westward-propagating MRG waves, using three-dimensionally high resolution simulations of the continuously stratified primitive equations and to rationalize afterward the vertical scale selection of the zonal jets which appeared in the simulations. Thus, in contrast to the earlier studies, our setup is based on the full primitive equations in a continuously stratified fluid and we attempt to address the nonlinear interactions between quite disparate vertical scales, with both low and very high vertical modes.

After a brief review of the waves of the equatorial $\beta$-plane primitive equations and of the MRG wave velocity field in sections 2 and 3, the known results concerning the destabilization of midlatitude barotropic Rossby waves are recalled in section 4. Numerical evidence of the destabilization of MRG waves, leading to zonal jet formation, is presented in section 5 for different types of model configurations, involving either a channel geometry or a closed basin geometry. Section 6 formulates a simplified model of the general problem of scale selection of zonal jets. We compare the predictions of the simplified model with the results of the numerical primitive equations simulations. The discussion in section 7 summarizes our present conclusions. The slower dynamics of resonant triads occurring during the destabilization of intermediate wavelength MRG waves is relegated to an appendix, its physics being less relevant for the formation of strong zonal jets.

\section{Equatorial primitive equations}

The equatorial $\beta$-plane primitive equations can be written as (e.g. Majda 2003)

$$
\begin{aligned}
\frac{d u}{d t}-f v+\frac{\partial P}{\partial x} & =0, \\
\frac{d v}{d t}+f u+\frac{\partial P}{\partial y} & =0, \\
\frac{\partial u}{\partial x}+\frac{\partial v}{\partial y}+\frac{\partial w}{\partial z} & =0, \\
\frac{d b}{d t}+N^{2} w & =0, \\
\frac{\partial P}{\partial z}-b & =0,
\end{aligned}
$$

where $(x, y, z)$ are the space coordinates, $(u, v, w)$ the zonal, meridional and vertical velocity components, $P$ pressure, $b$ buoyancy, $N$ the Brunt-Vaissala frequency, $f=\beta y$ and $\frac{d}{d t}=\frac{\partial}{\partial t}+u \frac{\partial}{\partial x}+v \frac{\partial}{\partial y}+w \frac{\partial}{\partial z}$.

Baroclinic vertical modes of integer mode number $m$ are introduced as solutions of

$$
\frac{\partial G_{m}}{\partial z}=-\frac{N^{2}}{c_{m}^{2}} \int_{-H}^{z} G_{m} d z, \quad \int_{-H}^{0} G_{m} d z=0,
$$

$H$ being the domain depth, using boundary conditions $\frac{\partial G_{m}}{\partial z}=0$ at $z=0,-H$. In the present work we shall only consider constant values of $N$ so that $c_{m}=\frac{N H}{\pi m}$. 
Expanding in vertical modes the above variables,

$$
\begin{array}{r}
(u, v, P)=\sum\left(u_{m}, v_{m}, P_{m}\right) G_{m}(z) \\
w=\sum w_{m} \int_{-H}^{z} G_{m}(z) d z \\
b=\sum b_{m} \frac{\partial G_{m}}{\partial z}
\end{array}
$$

linearizing the equations of motion (2.1) about the state of rest and eliminating variables other than $v$ yield the differential equation for vertical mode $m$,

$$
\frac{\partial}{\partial t}\left(\frac{\partial^{2} v_{m}}{\partial x^{2}}+\frac{\partial^{2} v_{m}}{\partial y^{2}}\right)-\frac{1}{c_{m}^{2}}\left(f^{2} \frac{\partial v_{m}}{\partial t}+\frac{\partial^{3} v_{m}}{\partial t^{3}}\right)+\beta \frac{\partial v_{m}}{\partial x}=0 .
$$

For a given vertical mode $m$ an expansion

$$
v_{m}=\sum_{r \geqslant 0} \tilde{v}_{r, m}(x, t) \mathcal{D}_{r, m}(y)
$$

in Hermite functions $\mathcal{D}_{r, m}(y)$ satisfying

$$
\frac{d^{2} \mathcal{D}_{r, m}}{d y^{2}}-\frac{f^{2}}{c_{m}^{2}} \mathcal{D}_{r, m}=-(2 r+1) \frac{\beta}{c_{m}} \mathcal{D}_{r, m}
$$

yields for zonally propagating plane waves proportional to exp $[i(k x-\omega t)]$ the dispersion relation

$$
k^{2}+\beta \frac{k}{\omega}-\frac{\omega^{2}}{c_{m}^{2}}+(2 r+1) \frac{\beta}{c_{m}}=0
$$

For $r=-1,0,1, \cdots$ the Kelvin, Mixed Rossby-gravity, Rossby and gravity free waves solutions can be obtained (Matsuno 1966). The dispersion relation for free equatorial waves is plotted in Figure 1 for the lowest values of $r$.

For the case of a MRG wave $(r=0)$, the dispersion relation for vertical mode $m$ and zonal wave number $k$ is

$$
\omega=\frac{1}{2} c_{m}\left(k+\sqrt{k^{2}+4 \lambda_{m}^{-2}}\right) ; \quad \lambda_{m}=\sqrt{\frac{c_{m}}{\beta}} .
$$

\section{Nondimensionalisation}

In the present study we shall focus upon the destabilization mechanisms of a moderate-amplitude free MRG wave of vertical wavenumber $m^{\star}=\frac{m \pi}{H}$, where $m$ is an integer, zonal wavenumber $k^{\star}$ and frequency $\omega^{\star}$. The Kelvin wave speed for the vertical mode of the basic state is denoted $c^{\star}$ and its baroclinic equatorial radius of deformation is $\lambda^{\star}=\sqrt{c^{\star} / \beta}$.

Hereafter the notations are such that dimensional quantities are denoted as ( $)^{\star}$ while nondimensional quantities have no particular annotation.

Independent variables are non-dimensionalized by

$$
x=x^{\star} / \lambda^{\star} ; \quad y=y^{\star} / \lambda^{\star} ; \quad z=z^{\star} m^{\star} ; \quad t=t^{\star} \sqrt{\beta c^{\star}},
$$

and velocities $(u, v, w)$ are scaled by $c^{\star}\left(1,1, \frac{1}{m^{\star} \lambda^{\star}}\right)$. The nondimensional basic state velocity components, 
relative vorticity and divergence are

$$
\left\{\begin{array}{rlll}
V & =\quad \mathcal{D}_{0,1}(y) & & \cos (k x-\omega t) \cos (z), \\
U & =-\omega \frac{1}{\sqrt{2}} \mathcal{D}_{1,1}(y) & & \sin (k x-\omega t) \cos (z), \\
W & =\omega^{2} \frac{1}{\sqrt{2}} \mathcal{D}_{1,1}(y) & & \cos (k x-\omega t) \sin (z), \\
\mathcal{Z} & =-k \quad\left(\mathcal{D}_{0,1}(y)+\frac{\omega}{k} \frac{\partial^{2}}{y^{2}} \mathcal{D}_{0,1}(y)\right) & \sin (k x-\omega t) \cos (z), \\
\frac{\partial W}{\partial z}=\omega^{2} \frac{1}{\sqrt{2}} \mathcal{D}_{1,1}(y) & & \cos (k x-\omega t) \cos (z),
\end{array}\right.
$$

where $k=k^{\star} \lambda^{\star}, \omega=\omega^{\star} / \sqrt{\beta c^{\star}}$,

$$
\mathcal{D}_{r, p}(y)=\frac{1}{\sqrt{2^{r} r ! \sqrt{\pi}}} H_{r}(\sqrt{p} y) \exp \left(-p \frac{y^{2}}{2}\right)
$$

is the $r t h$ Hermite function for the pth vertical harmonic of the basic state, and $H_{r}$ is the Hermite polynomial of order $r$.

The basic state can be written in dimensional form as

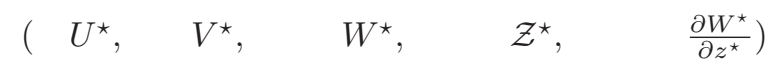

$$
\begin{aligned}
& =F r\left(c^{\star} U, \quad c^{\star} V, \quad \frac{c^{\star}}{m^{\star} \lambda^{\star}} W, \quad \sqrt{\beta c^{\star}} \mathcal{Z}, \quad \sqrt{\beta c^{\star}} \frac{\partial W}{\partial z}\right),
\end{aligned}
$$

where the variables $\left(U, V, W, \mathcal{Z}, \frac{\partial W}{\partial z}\right)$ are defined in (3.1) and we define the Froude number of the basic state as $F r \equiv \frac{V_{0}^{\star}}{c^{\star}}$ where $V_{0}^{\star}$ is the amplitude of the basic state meridional velocity field.

\section{Stability of midlatitude barotropic Rossby waves}

Gill (1974) showed that a midlatitude barotropic Rossby wave is always unstable and that the nature of the instability depends on the parameter $M=\frac{\mathcal{U} \kappa^{2}}{\beta}$, where $\kappa^{2}$ is the horizontal wavenumber squared and $\mathcal{U}$ the velocity scale of the Rossby wave. For large $M$, in particular in the limit of low wave frequency (since $\omega \sim \kappa^{-1}$ ), the problem reduces to the Rayleigh problem for a sinusoidal velocity distribution, involving the Rayleigh inflection point criterion for barotropic shear instability.

In Gill (1974) the perturbation stream function takes the form of a linear combination of a free barotropic Rossby wave, called the secondary wave, and its interactions with harmonics of the basic state Rossby wave, referred to as the primary wave. In the large $M$ limit, it is shown that for instability, the wavevector of the secondary wave of the perturbation can lie within a finite area of wavenumber space, and that the secondary wave of the most unstable perturbation has wavevector orthogonal to and of length comparable to that of the primary wave. For a Rossby wave with purely zonal wavenumber, the most unstable perturbation therefore contains a zonally symmetric component corresponding to a zonal jet structure with meridional scale comparable to the zonal wavelength of the basic state. Away from the large $M$ limit, the primary and secondary waves must form part of a resonant triad for instability, and as such the area of wavenumber space in which the secondary wavevector may lie is reduced to a curve. In that limit, the secondary wave of the most unstable perturbation does not have its wavevector orthogonal to that of the primary wave.

Given that in the large negative $k$ limit, the MRG wave dispersion relation approaches that of the 
midlatitude barotropic Rossby wave and that the motion is almost horizontally nondivergent in that limit, by extrapolating Gill's result one can expect that short westward MRG waves will destabilize through a barotropic instability mechanism and that their destabilization will give rise to large-scale zonal flows. On the other hand, the destabilization of long and intermediate wavelength westward MRG waves is likely to depend on triad interactions and not produce zonal flows. Eastward propagating MRG waves have relatively high frequencies, thus have little potential vorticity (since $P V \propto \frac{V^{\star}}{\omega^{\star}}$, see e.g. Ripa 1983), and are therefore not likely to lead to the large scale rearrangement of angular momentum necessary to produce zonal flows.

It is important to note that linear waves on the equatorial $\beta$-plane are not exact solutions of the nonlinear equations of motion, in contrast to linear quasi-geostrophic waves on the mid-latitude $\beta$-plane and linear internal waves, so the large $M$ limit also entails a large departure from the linear wave regime. We can nevertheless explore the large negative $k$ limit, independently of the wave amplitude. Also, trapped equatorial free waves have a baroclinic vertical structure which must be taken into account. We will see that the destabilization of a short MRG wave leads to a "barotropic" component, with vertical scales larger than or comparable to the primary wave, and a "baroclinic" component, with vertical scale determined by the vertical scale of free baroclinic equatorial waves with meridional scale comparable to the zonal scale of the basic state MRG wave.

\section{Numerical evidence of destabilization of Mixed Rossby Gravity waves}

The primitive equations code we have used for all the numerical simulations is ROMS (Shchepetkin \& McWilliams 2005). It is both highly parallelized and vectorized to efficiently run on the Earth Simulator, the usage of which was required for the high resolution simulations. For all cases the domain has a constant-depth $H=5000 \mathrm{~m}$, and the stratification is taken to be constant with $N=2 . \times 10^{-3} \mathrm{~s}^{-1}$. The basic state MRG wave is of low vertical mode, $m^{\star} \frac{H}{\pi}=2$, with a corresponding deformation scale of $2.3^{\circ}$ latitude. The meridional extent of the domain is $\left[-15^{\circ}, 15^{\circ}\right]$ latitude. The horizontal grid size is $1 / 11^{\circ}$ and the number of levels in the vertical varies between 200 and 400 to ensure a three-dimensionally consistent high resolution for capturing the EDJ-like structures. The explicit lateral dissipation and diffusion coefficients are $100 \mathrm{~ms}^{-2}$ while the vertical coefficients are $10^{-6} \mathrm{~ms}^{-2}$. Such small values for the vertical coefficients cannot affect the vertical scale selection of patterns in the numerical simulations.

\subsection{Periodic channel geometry simulations}

We have first performed initial-value simulations in a zonally-periodic channel geometry, with the basic state initialized as a free MRG wave. Simulations were performed for $k=-0.75,-1.5,-3,-4.5,-6$ (corresponding to the leftmost cross symbols in Figure 1). Such free waves have dimensional periods $T^{\star}$ of $17,24,40,56$ and 74 days and nondimensional frequencies $\omega=0.69,0.50,0.30,0.21,0.16$, respectively. The zonal extent of the channel is taken to be $10^{\circ}$ in longitude or equal to the basic state MRG wavelength if the latter is larger than $10^{\circ}$. The simulations were performed with a meridional Froude number $F r=0.2$. 
Moreover, for westward-propagating MRG waves, additional simulations varying the Froude number have been performed to better assess the different flow regimes.

Note that initial-value numerical simulations performed for eastward-propagating MRG waves (see the rightmost 2 cross symbols in Figure 1) reveal that their adjustment does not lead to the formation of low-frequency zonal flows and mostly involves the excitation of first harmonics of the initial wave, both in zonal wavenumber and vertical wavenumber (figures not shown). On the other hand, the westwardpropagating cases on which we focus lead to more interesting flows.

As the zonal wavenumber $k$ increases in magnitude, the MRG wave relative vorticity varies from being dominated by the term $-\frac{\partial U}{\partial y}$ in the long wave limit to being dominated by the term $\frac{\partial V}{\partial x}$ in the short wave limit. Since the ratio $\left(\frac{\partial V}{\partial x} /-\frac{\partial U}{\partial y}\right) \propto k / \omega$, the MRG wave destabilization for cases with $|k|<1$ will behave more like the zonally-symmetric regime $k=0$ of d'Orgeville \& Hua (2005), where the $\frac{\partial U}{\partial y}$ shear term is shown to control the growth rate of the instability. The case $k=-0.75$ (not shown) indeed differs little from the zonally-symmetric case, with barotropic westward motions at the equator and extra-equatorial eastward flow near the critical latitude $y_{c}^{\star}=\frac{\omega^{\star}}{2 \beta}$ (Figure 13 of d'Orgeville \& Hua 2005).

For values of $|k|>1$, the instantaneous zonal velocity distribution at a given longitude is shown after 150 days integration for $k=-1.5,-3,-4.5,-6$ in Figure 2(a)-(d). The integration of the intermediate wavelength case $k=-1.5$ has been pursued further in time and its interpretation in terms of resonant triads is discussed in Appendix A. At time $t^{\star}=150$ days, the basic state MRG wave has hardly been modified and the latitude-depth plot in Figure 2(a) only shows the antisymmetric zonal velocity field of a free MRG wave. For $k \leqslant-3$, the plots reveal that the rapid basic state wave destabilization leads to a significantly richer baroclinic flow field, with vertical scales that become smaller as the basic state wave becomes shorter (i.e. for increasing values of $|k|$ ). The depth-longitude sections of zonal velocity along the equator presented in Figure 2(e),(f) for the cases $k=-1.5,-3$ respectively, demonstrate that, of the two cases, the destabilization of the initial MRG wave leads to the formation of zonally-symmetric jet structures only for $k=-3$. For the very short wave limit (e.g. $k=-4.5,-6)$ the equivalent barotropic component (the harmonics $p=0$ and $p=2$ ) $\dagger$ of the flow reaches the largest amplitude among all vertical modes, with a strengthening of the extra-equatorial belt of eastward currents at $\pm 2^{\circ}$ latitude and with westward flow at the equator. This equivalent barotropic component dominates in amplitude the smaller vertical scales that are nevertheless still visible in the immediate vicinity of the equator and at $\pm 2^{\circ}$ latitude. The barotropic zonal jets retain a well-defined meridional scale comparable to the zonal scale of the MRG wave, whereas at the equator the baroclinic vertical scales become smaller and smaller with time. However, even without a clear vertical scale selection for each case, the vertical scales are smaller at a given nondimensional time for $k=-6$ than for $k=-4.5$.

\subsection{Simulations with localized initial conditions and oscillating forcing}

In order to shed light on the structures involved in the destabilisation process, simulations with two other configurations have been performed which allow for eastward and/or westward propagation of distur- 
bances away from the the instability region. The first alternative configuration is a basin geometry where the basic state MRG wave is forced by an oscillating source in the western boundary layer, confining the destabilization of the basic state to a western portion of the basin thereby enabling eastward propagating signals to be studied (d'Orgeville et al. 2007). The second is a zonally-localized basic state MRG wave is initialized in a very long channel so that eastward and westward propagating signals are more easily distinguished.

\subsubsection{Basin geometry}

In order to excite MRG waves in a basin, a baroclinic, temporally oscillating mass flux confined to the western boundary is applied at its northern and southern limits. The basin simulations are more thoroughly discussed in d'Orgeville et al. (2007) for a large set of simulations varying both $k$ and $m^{\star}$, and the interested reader is referred to that work for more details. The longitudinal extent of the basin is $30^{\circ}$. We present in figure 3(a)-(c) the zonal velocity along the equatorial cross section for the three cases $k=-3,-4.5,-6$ and $m^{\star} \frac{H}{\pi}=2$. We term these structures EDJ-like, in reference to the Equatorial Deep Jets that alternate with depth at the equator discussed in the papers cited in the introduction. These plots display a clear scale selection of zonal jets whose vertical modes are, respectively, around 6, 12 and 18 times smaller than the vertical mode of the basic state $m^{\star}$ for the three cases shown. In each case, the meridional scale of the jets is comparable to the zonal scale of the MRG wave. The confinement of the instability to the western part of the basin enables zonal jets alternating in sign in the vertical to develop in the rest of the basin, a result not observed in the channel geometry except for the case $k=-3$. The EDJ-like signal excited by the basic state MRG signal propagates eastward until it encounters the eastern boundary where it reflects to form a basin mode, the details of which are studied by d'Orgeville et al. (2007). Moreover, the EDJ vertical scale selection is reported there to be rather insensitive to the vertical mode $m^{\star}$ of the basic state wave, being primarily set by the dimensional period $T^{\star}$ of the basic state MRG wave. We shall return to this result in the next section, which attempts to provide a theoretical rationale for the vertical scale selection mechanism. For the basin configuration case, the low vertical modes remain confined to the western part of the basin, building up an interesting extra-equatorial meridional structure, plotted in Figure 3(d) for the case $k=-6$, with a quasi-barotropic belt of eastward currents at $\pm 2^{\circ}$ latitude flanking the EDJ-like structures confined to the equator.

\subsubsection{Zonally-localized $M R G$ wave}

The same three cases, $k=-3,-4.5,-6$ and $m^{\star} \frac{H}{\pi}=2$, have also been studied in a very long channel of zonal extent $150^{\circ}$, in which the basic state $\mathrm{MRG}$ wave is initially localized within $\left[40^{\circ}, 60^{\circ}\right]$ longitude with a Gaussian decay of $10^{\circ}$ at each end of the initialization subdomain. This initial-value problem allows both westward and eastward propagating signals to be distinguished and a more quantitative assessment of the vertical scale selection of the various types of jets.

For the cases $k=-3,-4.5,-6$, the eastward EDJ-like structure is found to be dominated by a vertical mode respectively 6,12 and 18 times higher than $m^{\star}$, the basic state MRG wave vertical mode, thus 
corroborating the observations of the basin geometry simulations. Therefore the vertical scale selection for the EDJ-like structures appears to be the same in the basin geometry and for the zonally-localized MRG wave.

We have opted to detail the simulation results for the $k=-6$ case in Figure 4. Instantaneous fields of the meridional and zonal velocity components along the equator after an integration of 330 days are presented in Figure 4(a) and 4(b) respectively. The short MRG wave basic state has a slow eastward group velocity and has moved eastward by less than $10^{\circ}$ after 330 days integration. In the longitude range where the basic state MRG wave, which dominates the meridional velocity field, is present, small vertical scales are triggered in zonal velocity. This is further corroborated by the zoom on the $\left[55^{\circ}, 75^{\circ}\right]$ longitude range of the zonal velocity field shown in Figure 4(d) which shows zonal jets of alternating direction in the vertical, and by the vertical mode spectrum of zonal velocity along the equator at the same instant presented in Figure $4(\mathrm{c})$. In the $\left[50^{\circ}, 70^{\circ}\right]$ longitude range, peaks are found at nondimensional vertical mode 18, corresponding to EDJ-like structure.

The extra-equatorial structure of the flow can be grasped from Figure 4(e) which shows a meridional section of zonal velocity at $60^{\circ}$ longitude, time-averaged over a period of the basic state MRG wave. A belt of eastward currents, approximately equally split between the barotropic component and harmonic $p=2$, at $\pm 2^{\circ}$ latitude flanks the EDJ-like signal which can be seen right at the equator. Note that as in the other simulations, the signal has a meridional scale comparable to the zonal scale of the initial wave.

Figure 5 presents Hovmöller plots for several harmonics. In Figure5(a), the barotropic mode signal is dominated by zonal velocity and it propagates westward faster than any baroclinic signal. At the final time, Figure 5(b) shows the meridional velocity of the MRG wave basic state with a westward phase propagation and an eastward group propagation of the eastward front of the initially-localized MRG wave. The response eastward of $60^{\circ}$ is dominated by very high harmonics and the largest amplitudes are found around $p=17$ for the meridional velocity component (Figure $5(\mathrm{c})$ ) $p=18$ for the zonal velocity component (Figure 5(d)). The $p=17$ signal presents the characteristics of a MRG wave disturbance of vertical wavenumber $17 m^{\star}$ while the $p=18$ signal corresponds to the highly baroclinic jets of Figure 4(d). The eastward propagation in Figure 5(d) fits very closely with a Kelvin wave of vertical wavenumber $18 m^{\star}$.

\subsection{Summary for zonally-short $M R G$ wave destabilization}

For large enough values of $|k|(>2)$, the westward phase propagating MRG wave strongly destabilizes leading to the formation of zonal jets. The characteristics of the zonal jets vary with the geometry of the experiment. In the periodic reentrant channel geometry, barotropic zonal jets dominate the total flow response at the equator and its immediate vicinity. For simulations with localized initial conditions or oscillating forcing, the destabilization leads to quite different characteristics of the zonal jets for eastward propagating signals. The most striking result concerns the formation of zonal jets alternating in sign in the vertical, with quite high baroclinic mode and located right at the equator. Such characteristics are observed both in the initial-value, zonally-localized MRG wave simulations and in the basin simulations 
with a forcing of MRG wave signals in the western boundary layer. For the same period of the MRG -wave basic state, these two cases yield the same vertical mode selection for the baroclinic equatorial jets.

Independent of the domain geometry, the meridional scales of both barotropic and baroclinic zonal jets are comparable to the zonal scale of the MRG wave basic state $\dagger$.

$\dagger$ We stress that latitude-longitude plots of the velocity field components and Ertel potential vorticity (not shown) during the destabilization process of our short, balanced, westward-propagating MRG wave basic state involve no chevron-like structures, such as those seen by Bouchut et al. (2005) for the breaking through shock formation of short, unbalanced, eastward-propagating MRG waves of finite-amplitude. 


\section{A simplified model of MRG wave destabilization}

The general objective of this section is to formulate a simplified model that can shed light on (i) the vertical scale selection of the baroclinic zonal jets of section 5.2, (ii) the meridional pattern of barotropic zonal jets of section 5.1, and (iii) the scaling of growth rates for both types of jets as a function of the characteristics of the basic state MRG wave.

Our physical context is similar to that of Gill (1974) but has significant differences. An obvious similarity is that the basic state MRG wave has zonal wavelength and frequency characteristics close to those of a short midlatitude barotropic Rossby wave. The fastest growing disturbances observed in the numerical simulations also have characteristics similar to those predicted by Gill, most notably that the meridional scale of disturbances is as short as the zonal scale of the basic state wave. In a stratified rotating flow, there are two ways of obtaining short meridional scales: either through low meridional modes of high vertical modes (section 6.2) or through high meridional modes of low vertical modes (section 6.3).

An important difference from the Gill problem is that we are dealing with a stratified fluid, and this introduces a vertical dimension to the problem, allowing for the possibility of both baroclinic and barotropic zonal jets to coexist. In which situation will a given structure prevail? We shall see that the formulation of the baroclinic zonal jets problem involves free baroclinic equatorial waves and thus requires a representation in Hermite functions in $y$, while the non-divergent barotropic zonal jets problem involves disturbances that are sinusoidal in both $x$ and $y$. Thus a different mathematical treatment is demanded for each case.

We summarize the assumptions used in our simplified model of short MRG wave destabilization: Gill's treatment of the barotropic vorticity equation is of Galerkin type, constructing a solution out of waves whose zonal wavenumbers are multiples of that of the basic state, including zero, i.e. a set of spatial structures corresponding to low-frequency free waves of the barotropic system. We too are concerned with low-frequency motion, and therefore construct the spatial structure of our solution from the spatial structures of the low-frequency equatorial free waves, both long and short. Like in Gill (1974), we shall restrict ourselves to a Galerkin representation of the low-frequency vorticity equation. Our approach thus ignores the prognostic divergence equation, but the dynamics of low-frequency "vortical" waves is dominated by vorticity physics, divergence effects coming into play only through the diagnostic vortex stretching term. The vorticity equation is linearized about the zonally short basic state of wavenumber $k$, discarding terms of higher order in Froude number. Scalings of perturbations and of the short MRG wave basic state are performed to further simplify the vorticity equation. The $4 \mathrm{D}$ problem for a given MRG wave basic state is projected onto an appropriate basis of functions in $x, y, z$ and $t$ in order to arrive at an eigenvalue problem with constant real coefficients for both the baroclinic and barotropic zonal jets . We stress that the solution is composed of forced waves.

The remainder of this section is organized as follows. The subsection 6.1 presents the equations and the scaling used in the analysis. The cases of the baroclinic and barotropic jets are treated in subsections 
6.2 and 6.3 respectively. The underlying physical mechanism of the instability is discussed in subsection 6.4 .

\subsection{Scalings for linearized vorticity equation}

The zonally-short wave limit of the dispersion relation of westward-propagating MRG waves is known to be similar to that of the barotropic Rossby wave (Matsuno 1966). Both types of waves have horizontally non-divergent motions. Figure 1 shows that for all westward propagating MRG waves, $\omega<1$ and $\omega \longrightarrow 0$ as $k \longrightarrow-\infty$. The non-dimensional MRG wave dispersion relation

$$
\omega=\frac{1}{\omega-k}
$$

approaches the nondimensional barotropic Rossby wave dispersion relation for zonally propagating waves, $\omega=-\frac{1}{k}$ (dashed line in Figure 1), as $k \longrightarrow-\infty$. The small parameter which is used in the analysis given below is based on $\omega \sim k^{-1} \ll 1$.

Momentarily dropping * symbols for dimensional quantities in the next equation, the full dimensional vorticity equation for $\zeta \equiv \frac{\partial v}{\partial x}-\frac{\partial u}{\partial y}$ is

$$
\left(\frac{\partial}{\partial t}+u \frac{\partial}{\partial x}+v \frac{\partial}{\partial y}+w \frac{\partial}{\partial z}\right)(\zeta+f)-(\zeta+f) \frac{\partial w}{\partial z}+\frac{\partial w}{\partial x} \frac{\partial v}{\partial z}-\frac{\partial w}{\partial y} \frac{\partial u}{\partial z}=0 .
$$

Its linearization and nondimensionalisation yield (denoting by primes the disturbances to the MRG wave basic state)

$$
\begin{gathered}
\frac{\partial \zeta^{\prime}}{\partial t}+v^{\prime}-y \frac{\partial w^{\prime}}{\partial z} \\
+F r\left\{\left(U \frac{\partial}{\partial x}+V \frac{\partial}{\partial y}+W \frac{\partial}{\partial z}\right) \zeta^{\prime}+\left(u^{\prime} \frac{\partial}{\partial x}+v^{\prime} \frac{\partial}{\partial y}+w^{\prime} \frac{\partial}{\partial z}\right) \mathcal{Z}\right. \\
\left.-\mathcal{Z} \frac{\partial w^{\prime}}{\partial z}-\zeta^{\prime} \frac{\partial W}{\partial z}+\frac{\partial W}{\partial x} \frac{\partial v^{\prime}}{\partial z}+\frac{\partial w^{\prime}}{\partial x} \frac{\partial V}{\partial z}-\frac{\partial W}{\partial y} \frac{\partial u^{\prime}}{\partial z}-\frac{\partial w^{\prime}}{\partial y} \frac{\partial U}{\partial z}\right\}=\mathcal{O}\left(F r^{2}\right),
\end{gathered}
$$

where the curly bracket terms are all of order Fr. Self-interaction terms of disturbances and selfinteraction terms of the basic state MRG wave are neglected. The latter are nonzero because unlike the case of a midlatitude Rossby wave, a MRG wave is not an exact solution of the nonlinear equations of motion. Such terms are of order $\mathrm{Fr}^{2}$ and moreover project only on the barotropic mode and the first harmonic of the basic state, and as such are neglected in the following analysis $†$.

\subsubsection{Basic state and disturbances scaling}

Numerical simulations reveal the formation of two different types of zonal jets: barotropic zonal jets for the periodic channel simulations and high vertical mode baroclinic jets for the other cases. Likewise, our analysis of growing disturbances is split into two cases: baroclinic horizontally divergent disturbances and horizontally non-divergent "barotropic" disturbances (sections 6.2 and 6.3 respectively). However, a general scaling of the various types of perturbations appropriate to both cases can be performed.

Equation (6.2) has coefficients that are periodic in $x$ with period $2 \pi / k$. Bounded solutions will have

$\dagger$ It can be shown that the ratio of the largest term in $F r^{2}$ and the largest term in $F r$ is in $\frac{F r}{k}$, which is small for large $k$ and $\mathrm{Fr}<1$. 
the same periodicity and we thus assume $\partial_{x} \sim k$. Gill (1974) showed that the meridional scale of the most unstable disturbance is comparable to the zonal scale of the zonally-short Rossby wave, a scaling that is corroborated by our simulations. Hence, we take $\partial_{y} \sim k$. The vertical scale is related to the meridional through the equatorial radius of deformation for vertical mode $m, \sqrt{N H / m \beta}$, whereby motions of nondimensional meridional scale $\sim 1 / k$ have nondimensional vertical scale $\sim 1 / k^{2}$. We therefore take $\partial_{z} \sim k^{2}$ for the divergent disturbances.

Since both zonal and meridional components of the velocity disturbance involve comparable spatial scales, the horizontal velocity scaling is $v \sim u \sim 1$. Divergent baroclinic disturbances require $\frac{\partial w}{\partial z}=$ $-\left(\frac{\partial u}{\partial x}+\frac{\partial v}{\partial y}\right) \sim k$, whence $w \sim k^{-1}$.

From (3.1), in the limit $|k| \gg 1$, and in the neighbourhood of the equator where the expected dominant baroclinic modes are trapped, that is where $y \sim O\left(k^{-1}\right)$, the basic state MRG wave fields scale like

$$
\begin{aligned}
(U, V, W) \sim\left(\frac{\omega}{k}, 1, \frac{\omega^{2}}{k}\right) & \sim\left(k^{-2}, 1, k^{-3}\right), \\
\left(\mathcal{Z}, \frac{\partial W}{\partial z}\right) \sim\left(k, \frac{\omega^{2}}{k}\right) & \sim\left(k, k^{-3}\right),
\end{aligned}
$$

and note that the rescaling of the spatial derivatives applies only to the perturbation fields and not to the basic state.

\subsection{Baroclinic zonal jets}

We explicitly inject the scalings of the previous section for both the zonally-short basic state MRG wave and for the baroclinic disturbances of high vertical mode into the linearized vorticity equation (6.2). This is detailed in Appendix B to show that the system reduces to

$$
\frac{\partial \zeta^{\prime}}{\partial t}+v^{\prime}-y \frac{\partial w^{\prime}}{\partial z}+F r\left\{V \frac{\partial \zeta^{\prime}}{\partial y}+u^{\prime} \frac{\partial \mathcal{Z}}{\partial x}-\mathcal{Z} \frac{\partial w^{\prime}}{\partial z}\right\}=0,
$$

where the zonally-short basic state has nondimensional variables satisfying

$$
\left\{\begin{array}{rr}
V= & \cos \theta \cos z D_{0,1}(y), \\
\mathcal{Z} \approx \frac{\partial V}{\partial x}= & -k \sin \theta \cos z D_{0,1}(y), \\
\frac{\partial \mathcal{Z}}{\partial x} \approx \frac{\partial^{2} V}{\partial x^{2}}= & -k^{2} \cos \theta \cos z D_{0,1}(y),
\end{array}\right.
$$

with $\theta \equiv k x-\omega t$ and where terms in $O\left(\omega^{2}\right)$ in $\mathcal{Z}$ have been neglected. This leads to

$$
\frac{\partial}{\partial t} \zeta^{\prime}+v^{\prime}-y \frac{\partial w^{\prime}}{\partial z}+F r \cos z D_{0,1}(y)\left\{\cos \theta\left(\frac{\partial \zeta^{\prime}}{\partial y}-k^{2} u^{\prime}\right)+\sin \theta k \frac{\partial w^{\prime}}{\partial z}\right\}=0 .
$$

Changing to the new set of independent variables $(\theta, y, z, t)$, equation (6.4) transforms to

$$
\frac{\partial}{\partial t} \zeta^{\prime}-\omega \frac{\partial}{\partial \theta} \zeta^{\prime}+v^{\prime}-y \frac{\partial w^{\prime}}{\partial z}+F r \cos z D_{0,1}(y)\left\{\cos \theta\left(\frac{\partial \zeta^{\prime}}{\partial y}-k^{2} u^{\prime}\right)+\sin \theta k \frac{\partial w^{\prime}}{\partial z}\right\}=0 .
$$

Motivated by the basin simulations of MRG waves forced from the western boundary and by the channel simulations with an initial zonally-localized MRG wave (section 5.2), our purpose is to determine what sets the vertical scale of the high baroclinic zonal jets that propagate outside of the forcing region where the basic state MRG wave is not present. We thus (i) seek an approximate solution to the perturbation vorticity equation as a linear combination of low-frequency free equatorial wave spatial structures, and 
(ii) restrict our solution to structures of waves with eastward group velocities. Concerning point (i), our choice of solution in terms of free wave structures ensures that the unstable modes will correspond to structures observed outside of the forcing region. Concerning point (ii), since we are primarily interested in the signal that develops eastward of the zonally-localized MRG wave and of the forcing in the basin simulations, the expansion in free waves is restricted to baroclinic free waves which have eastward group velocities. Both approximations are justified a posteriori by comparing our theoretical predictions to the numerical simulations.

Introducing the vector of velocity disturbances $\underline{X^{\prime}} \equiv\left(\begin{array}{c}u^{\prime} \\ v^{\prime}\end{array}\right)$, the relative vorticity $\zeta^{\prime}$ and divergence $\delta^{\prime} \equiv-\frac{\partial w^{\prime}}{\partial z}$ are

$$
\left(\begin{array}{c}
\zeta^{\prime} \\
\delta^{\prime}
\end{array}\right)=\left(\begin{array}{c}
\underline{\hat{z}} \cdot\left(\nabla_{H} \times \underline{X^{\prime}}\right) \\
\nabla_{H} \cdot \underline{X}^{\prime}
\end{array}\right)
$$

where $\underline{\hat{z}}$ is the unit vector in the vertical direction and $\nabla_{H} \equiv\left(\frac{\partial}{\partial x}, \frac{\partial}{\partial y}\right)=\left(k \frac{\partial}{\partial \theta}, \frac{\partial}{\partial y}\right)$. Equation (6.5) is of the form

$$
\frac{\partial}{\partial t} \mathcal{L}(\theta, y) \underline{X}^{\prime}+\mathcal{M}(\theta, y, z) \underline{X}^{\prime}=0
$$

where $\mathcal{L}(\theta, y)$ and $\mathcal{M}(\theta, y, z)$ are linear differential operators that are independent of $t$. Thus the solution is of the form

$$
\underline{X}^{\prime}(\theta, y, z, t)=\mathcal{R}\{\exp (\mu t) \underline{\tilde{X}}(\theta, y, z)\}
$$

where $\mu$ is complex and $\mathcal{R}$ denotes the real part.

The next step for solving the system is the successive elimination of $\theta, z$ and $y$, which is described in the next subsection 6.2.1. This leads to an eigenvalue problem of the form

$$
[E] \underline{Y}=\mu \underline{Y},
$$

where the unknown vector $\underline{Y}$ is composed of the amplitude coefficients of the long and short waves which interact with the primary wave.

The spatial structure of the long waves is that of a Kelvin wave, because of the eastward group velocity requirement, while the short waves are MRG and Rossby waves. We emphasize that the solution is composed of forced waves, the frequencies of which are determined by the basic state and the vertical modes that are selected.

The casual reader may safely skip the next subsection, the solution of the eigenvalue problem (6.8) being summarized in section 6.2 .2 and Figure 6 .

\subsubsection{Elimination of $\theta, y$ and $z$ leading to the matrix eigenvalue problem}

$\tilde{u}, \tilde{v}, \tilde{\zeta}, \tilde{\delta}$ are used below to denote the parts of disturbance fields that are functions of $(\theta, y, z)$.

- Elimination of $\theta$

Equation (6.6) is a second order differential equation in $\theta$ for $\underline{\tilde{X}}$ with periodic coefficients that are invariant under $\theta \longrightarrow \theta+2 \pi$. We seek a solution with the same periodicity in $\theta$, which can therefore be represented 
as a Fourier series

$$
\underline{\tilde{X}}=\sum_{n} \underline{\tilde{X}}_{n} \exp i n \theta
$$

where $i^{2}=-1$.

For mid-latitude barotropic Rossby waves, the phase $\theta$ is a function of $x, y$ and $t$, while for the case of equatorial waves the propagation is only zonal so that $\theta$ is only a function of $x$ and $t$. Our focus here is on the formation of zonal jets, which are represented by the Fourier component $\underline{\tilde{X}}_{0}$ corresponding to a signal that does not vary in $x$. For instance, velocity disturbances of the form $\underline{X^{\prime}}=\exp (\mu t)\left(\begin{array}{c}\tilde{u}(y) \\ 0\end{array}\right)$ represent a zonally-invariant zonal jet, corresponding to the long wave limit of a growing long free wave of very low frequency.

As in Gill (1974) we shall truncate the Fourier series to $n=-1,0,+1$ :

$$
\begin{aligned}
\underline{\tilde{X}} & =\underline{X}_{L}(y, z)+\underline{X}_{S}^{+}(y, z) \exp i \theta+\underline{X}_{S}^{-}(y, z) \exp (-i \theta) \\
& =\left(\begin{array}{c}
u_{L} \\
0
\end{array}\right)+\left(\begin{array}{c}
u_{S}^{+} \\
-i v_{S}^{+}
\end{array}\right) \exp i \theta+\left(\begin{array}{c}
u_{S}^{-} \\
i v_{S}^{-}
\end{array}\right) \exp (-i \theta)
\end{aligned}
$$

where $u_{L}, u_{S}^{ \pm}$and $v_{S}^{ \pm}$are complex functions of $(y, z)$. The subscript $S$ stands for short waves with the same dimensional wavenumber $k^{\star}$ as the basic state, the subscript $L$ stands for long waves, and we shall use the notations

$$
\left(\begin{array}{c}
\tilde{\zeta} \\
\tilde{\delta}
\end{array}\right)=\left(\begin{array}{c}
\zeta_{L} \\
0
\end{array}\right)+\left(\begin{array}{c}
\zeta_{S}^{+} \\
-i \delta_{S}^{+}
\end{array}\right) \exp i \theta+\left(\begin{array}{c}
\zeta_{S}^{-} \\
i \delta_{S}^{-}
\end{array}\right) \exp (-i \theta)
$$

where the $\zeta_{L}, \zeta_{S}^{ \pm}, \delta_{S}^{ \pm}$are complex functions of $(y, z)$.

Projecting (6.5) onto exp $i \theta$, exp $-i \theta$ and 1 yields

$$
\begin{cases}(\mu-i \omega) \zeta_{S}^{+}-i\left(v_{S}^{+}+y \delta_{S}^{+}\right) & +F r \cos z D_{0,1}(y)\left\{\frac{\partial \zeta_{L}}{\partial y}-k^{2} u_{L}\right\}=0 \\ (\mu+i \omega) \zeta_{S}^{-}+i\left(v_{S}^{-}+y \delta_{S}^{-}\right) & +F r \cos z D_{0,1}(y)\left\{\frac{\partial \zeta_{L}}{\partial y}-k^{2} u_{L}\right\}=0 \\ \mu \zeta_{L} & +\frac{F r}{2} \cos z D_{0,1}(y)\left\{\frac{\partial\left(\zeta_{S}^{+}+\zeta_{S}^{-}\right)}{\partial y}-k^{2}\left(u_{S}^{+}+u_{S}^{-}\right)-k\left(\delta_{S}^{+}+\delta_{S}^{-}\right)\right\}=0\end{cases}
$$

- Elimination of $z$

An expansion of $\underline{X}_{L}$ and $\underline{X}_{S}^{ \pm}$into vertical harmonics of the basic state is used:

$$
\begin{aligned}
& \underline{X}_{L}(y, z)=\sum_{p} \cos p z \underline{X}_{L, p}(y), \\
& \underline{X}_{S}^{ \pm}(y, z)=\sum_{p} \cos p z \underline{X}_{S, p}^{ \pm}(y),
\end{aligned}
$$

from which vorticity and divergence can be computed for each $p$.

By projecting (6.11) onto $\cos p z$ we get

$$
\left\{\begin{array}{lrl}
(\mu \mp i \omega) \zeta_{S, p}^{ \pm}(y) \mp i\left(v_{S, p}^{ \pm}(y)+y \delta_{S, p}^{ \pm}(y)\right) & +\frac{F r}{2} D_{0,1}(y)\left[\Lambda_{S, p+1}(y)+\Lambda_{S, p-1}(y)\right]=0 \\
\mu \zeta_{L, p}(y) & +\frac{F r}{4} D_{0,1}(y)\left[\Lambda_{L, p+1}(y)+\Lambda_{L, p-1}(y)\right]=0
\end{array}\right.
$$


where

$$
\left\{\begin{aligned}
\Lambda_{S, p}(y) & \equiv \frac{\partial \zeta_{L, p}}{\partial y}-k^{2} u_{L, p}, \\
\Lambda_{L, p}(y) & \equiv \frac{\partial\left(\zeta_{S, p}^{+}+\zeta_{S, p}^{-}\right)}{\partial y}-k^{2}\left(u_{S, p}^{+}+u_{S, p}^{-}\right)-k\left(\delta_{S, p}^{+}+\delta_{S, p}^{-}\right) .
\end{aligned}\right.
$$

- Elimination of $y$

For a given vertical harmonic $p$, the meridional structure of $\underline{X}_{L, p}(y)$ and $\underline{X}_{S, p}^{ \pm}(y)$ is found through an expansion in the meridional structures of low-frequency, free equatorial waves with eastward group velocities, and, for the short wave component, an identical zonal wavenumber $k$ to that of the basic state MRG wave.

The long waves correspond to long Kelvin wave disturbances. For the short waves, MRG and short Rossby waves with eastward group velocity are included. Thus, let

$$
\left\{\begin{array}{c}
\underline{X}_{L, p}(y)=\hat{u}_{L, p, 0}\left(\begin{array}{c}
D_{0, p}(y) \\
0
\end{array}\right), \\
\underline{X}_{S, p}^{ \pm}(y)=\sum_{\substack{0 \leqslant r \leqslant r_{\max }(p) \\
r \text { even }}} \hat{v}_{S, p, r}^{ \pm}\left(\begin{array}{c}
\frac{\sqrt{p}}{2}\left[\frac{\sqrt{2(r+1)}}{p \omega_{p, r}^{ \pm} \mp} D_{r+1, p}(y)+\frac{\sqrt{2 r}}{p \omega_{p, r}^{ \pm} \pm} D_{r-1, p}(y)\right] \\
\mp i D_{r, p}(y)
\end{array}\right),
\end{array}\right.
$$

where $\omega_{p, r}^{+}=-\omega_{p, r}^{-}$is a solution of the free equatorial wave dispersion relation (2.4) for the same dimensional zonal wavenumber as that of the basic state, an integer vertical mode number $\mathrm{pm}$ and a meridional mode number $r$. Note that we use the notation $\hat{u}_{L, p, 0}$ because the Hermite function is of order zero for the zonal velocity of the Kelvin wave.

The short waves are MRG and short Rossby waves of even meridional mode number $r$ (so that the zonal velocity is symmetric in $y) \cdot r_{\max }(p)$ corresponds to the short Rossby wave with zero group velocity at given $k^{\star}$. The zero group velocity for Rossby waves is obtained for

$$
k^{\star}=-\left[\frac{p(2 r+1) \beta}{c}\right]^{1 / 2} \quad \Longleftrightarrow k=-[p(2 r+1)]^{1 / 2}
$$

(cf. Gill (1982) p.445) and thus $r_{\max }(p)=\frac{1}{2}\left(\frac{k^{2}}{p}-1\right)$.

We make use of the property of free baroclinic waves that

$$
i\left(v_{S, p, r}^{ \pm}+y \delta_{S, p, r}^{ \pm}\right)=-i \omega_{p, r}^{ \pm} \zeta_{S, p, r}^{ \pm}
$$

and denote the frequency shift due to the difference between the forced frequency $\omega$ and the free wave frequency by

$$
\breve{\omega}_{p, r} \equiv \omega-\omega_{p, r}^{+}
$$

Injecting (6.15) into (6.13) gives 


$$
\left\{\begin{aligned}
\sum_{\substack{0 \leqslant r \leqslant r_{\max }(p) \\
r \text { even }}}\left(\mu \mp i \breve{\omega}_{p, r}\right) \zeta_{S, p, r}^{ \pm}(y) & +\frac{F r}{2} D_{0,1}(y)\left[\Lambda_{S, p+1,0}(y)+\Lambda_{S, p-1,0}(y)\right]=0, \\
\mu \zeta_{L, p, 0}(y) & +\frac{F r}{4} D_{0,1}(y)\left[\sum_{\substack{r \leqslant r_{\max }(p) \\
r \text { even }}}\left(\Lambda_{L, p+1, r}(y)+\Lambda_{L, p-1, r}(y)\right)\right]=0,
\end{aligned}\right.
$$

where $\Lambda_{L, p, r}$ and $\Lambda_{S, p, 0}$ are the projections of $\Lambda_{L, p}$ and $\Lambda_{S, p}$ onto meridional modes $r$ and 0 respectively. The resolution of (6.16) is performed by multiplying by the $y$-dependence of $\zeta_{S, p, r}(y)^{ \pm}$and $\zeta_{L, p, 0}(y)$ respectively and integrating in $y$ from $-\infty$ to $\infty$.

This leads to the eigenvalue problem (6.8), where the unknown vector $\underline{Y}$ is composed of $\hat{u}_{L, p, 0}$ for $p$ even and $\hat{v}_{S, p, r}^{ \pm}$for $p$ odd, and $[E]$ is a square matrix with constant real elements. For a vertical mode expansion such that $p \leqslant p_{\max }$, the length of $\underline{Y}$ is $\sum_{p=1}^{p_{\max }} r_{\max }(p)+\frac{p_{\max }}{2}$.

\subsubsection{Vertical scale selection}

System (6.8) has been solved for the values of $k$ corresponding to the numerical simulations described in section 5.2, with $p_{\max } \sim 100$, implying an eigenvalue problem of about $135 \times 135$.

The vertical scale selection is assessed by computing the vertical spectrum of the zonal velocity field of the most unstable eigenmode at the equator. Figure 6a obtained for a large set of $k$ values and for both $F r=0.1$ and $F r=0.2$ confirms that

$$
\mu \propto F r|k|,
$$

a result expected based on our scalings and the form of (6.5) (this is detailed in Appendix B). Furthermore, Figure 6a shows that the vertical mode numbers of the peaks of Figure $6 \mathrm{~b}$ satisfy

$$
p_{\text {peak }} \approx 0.55 k^{2} .
$$

Thus, as observed in the numerical simulations, the instability leading to EDJ-like structures is favoured by short basic state MRG waves. Specifically, peaks in the vertical harmonics spectra are found at $p_{\text {peak }}=6,12,20$ for $k=-3,-4.5,-6$, respectively (Figure $6 \mathrm{~b}$ ). The threshold beyond which positive growth rates are found for $p>2$ is around $|k|=2$. These theoretical findings demonstrate that there is a clear vertical scale selection for the formation of baroclinic zonal jets through the destabilisation of the basic state. Higher vertical scale baroclinic zonal jets are favoured by shorter MRG wave basic states.

The theoretical prediction can be quantitatively compared with the case $k=-6$ of the initially zonallylocalized MRG wave in a long channel simulation (section 5.2.2). An overall view of the various structures that are selected can be seen from the time-mean vertical mode spectrum computed at $x^{\star}=60^{\circ}$. This longitude is in the eastern portion of the interval where the MRG wave was initialized. Figure 7 presents three peaks in the vertical spectrum, at $p=18, p=6$ and $p=0$. Note that the peak in EDJs for the numerical simulation is at $p=18$ rather than at $p=20$ as predicted theoretically (Figure 7 ), but the peak 
for the numerical simulation is not very pronounced and the largest growth rates are centered around $p=20$. Time series of the amplitudes of the $p=20$ vertical harmonic of zonal velocity and the $p=19,21$ vertical harmonics of meridional velocity from the simulations are presented in Figures 8(a) and 8(b) respectively. The oscillations time scale seen in the figure are comparable to the dimensional period of the basic state $M R G$-wave for this case $\left(T^{\star}=74\right.$ days $)$. The time series provide evidence of exponential growth rates with values quite close to one another, thus justifying equation (6.7) where a collective growth rate for the superposition of different vertical modes is expected. The meridional structure of simulated zonal velocity for $p=20$ (dashed line) in Figure 8(c) moreover agrees with the most unstable Kelvin wave structure predicted by the theory (continuous line).

The most important result of our simplified model for the vertical scale selection for EDJ-like structures is thus that the characteristic dimensional vertical mode $p^{\star} \equiv p m^{\star}$ obeys

$$
p^{\star} \sim \frac{\beta N}{2 \omega^{\star 2}} \propto T^{\star 2}
$$

where $T^{\star} \equiv \frac{2 \pi}{\omega^{\star}}$ is the dimensional period of the basic state, and where we have used $\omega^{\star} \sim \frac{\beta}{k^{\star}}$. The longer the dimensional period of the basic state MRG wave, the higher the vertical mode of the excited EDJ-like structures, and the vertical mode number increases as the square of the MRG wave period. This prediction has been checked in the basin simulations of d'Orgeville et al. (2007) (their Figure 9), where forcings with the same dimensional period and different vertical wavenumbers $m^{\star}$ inside the western boundary layer yield the same vertical mode selection for the EDJ-like structures excited in the basin interior. Such a square law dependence is linked to the equatorial radius of deformation decrease as the square root of the vertical mode number.

\subsection{Non-divergent zonal jets}

The scale analysis of the linearized vorticity equation yields in this case

$$
\frac{\partial \zeta^{\prime}}{\partial t}+v^{\prime}+\operatorname{Fr}\left(V \frac{\partial \zeta^{\prime}}{\partial y}+u^{\prime} \frac{\partial \mathcal{Z}}{\partial x}\right)=0
$$

which written in terms of the new independent variables $(\theta, y, z, t)$ becomes

$$
\frac{\partial}{\partial t} \zeta^{\prime}-\omega \frac{\partial}{\partial \theta} \zeta^{\prime}+v^{\prime}+F r \cos z D_{0,1}(y)\left\{\cos \theta\left(\frac{\partial \zeta^{\prime}}{\partial y}-k^{2} u^{\prime}\right)\right\}=0
$$

Vertically-modulated perturbations involving barotropic modes are sought of the form

$$
\underline{X}^{\prime}=\mathcal{R}\{\exp (\mu(z) t) \underline{\tilde{X}}(\theta, y)\}
$$

where $\underline{\tilde{X}}(\theta, y)$ is periodic in $\theta$ with period $2 \pi$. The $z$ dependence in equation $(6.21)$ can be removed by choosing

$$
\mu=\tilde{\mu} \cos (z) .
$$

The elimination of the $\theta$ dependence closely follows the baroclinic case treatment (keeping only the $n=-1,0,1$ terms of the Fourier series) and is not repeated here. 
Equation (6.21) becomes

$$
\left\{\begin{array}{l}
(\tilde{\mu}-i \omega) \zeta_{S}^{ \pm} \mp i v_{S}^{ \pm}+\operatorname{Fr} \frac{1}{2} D_{0,1}(y)\left\{\frac{\partial \zeta_{L}}{\partial y}-k^{2} u_{L}\right\}=0 \\
\tilde{\mu} \zeta_{L}+\operatorname{Fr} \frac{1}{2} D_{0,1}(y)\left\{\frac{\partial \zeta_{S}^{+}+\zeta_{S}^{-}}{\partial y}-k^{2}\left(u_{S}^{+}+u_{S}^{-}\right)\right\}=0
\end{array}\right.
$$

This system is solved by projection onto free barotropic waves structures to find both the growth rate and meridional structure of the most unstable barotropic zonal jet. Specifically, the long barotropic wave has a sinusoidal dependence in $y$ only, while the short barotropic free wave has a $\cos \theta \operatorname{dependence~and~a~}$ sinusoidal dependence in $y$, with the appropriate mode quantization imposed by the channel geometry.

Figure 9 shows the meridional structure of the barotropic mode $p=0$ (dashed lines) during the early growth phase for the simulations performed in the periodic channel geometry (section 5.1) for a basic state MRG wave with $F r=0.2$ and $k=-3,-4.5,-6$. For Figures $9(\mathrm{~b})$ and $9(\mathrm{c})$ the meridional structure of the theoretically predicted most unstable eigenmode has also been plotted (continuous line), and shows an improving agreement with the simulations as $|k|$ becomes larger. As the MRG wave basic state becomes zonally shorter, the characteristic meridional width of the growing zonal jets scales as $k^{-1}$ and the growth rate varies as $\mu \propto F r k$, as already found for the baroclinic zonal jets (sections 6.2 and 6.2.2). For not too large values of $k$, the quantitative influence of the frequency shift term $-i \omega \zeta_{S}^{ \pm} \mp i v_{S}^{ \pm}$in (6.22) on the characteristics of the growing disturbances will be stronger for smaller $F r$. At $F r=0.2$ and $k=-3$ for instance, these terms cause the equatorially-trapped eigenmode (continuous line of Figure 9(a)) to have a smaller growth rate than other eigenmodes with additional extra-equatorial extrema (not shown). This may explain the less pronounced strength of the equatorially-trapped barotropic signal, enabling the higher baroclinic zonal jets with $p$ between 4 and 6 to dominate the final state (Figure 2(b)).

Note furthermore that the simulations always exhibit barotropic westward flow at the equator, as also found in d'Orgeville \& Hua (2005), while the theoretical unstable linear modes cannot predict any sign bias. Such issues of angular momentum mixing along with the documentation of the destabilization regimes of MRG waves with much higher $|k|$ will be presented in another forthcoming study (Fruman et al. (2008)).

Finally, the theoretical model (6.22) also predicts that the growth rate of the equivalent "barotropic" zonal jet has a vertical structure in $|\cos z|$. This is supported, for instance, by the channel simulations for $k=-4.5$ and $k=-6$ (Figures $2(\mathrm{c})$ and $2(\mathrm{~d})$ ), where the amplitude of the zonal velocity does not change sign with depth but has a depth-varying amplitude in $|\cos z|$, hence the quotation marks on the word "barotropic".

Eigensolutions of equation (6.22) are valid for all non-divergent disturbances and as such predict the common meridional profile of barotropic disturbances and of low vertical mode, almost non-divergent disturbances (see section 6.1.1). This explains why in the numerical simulations the meridional structures of the barotropic $(p=0)$ and harmonic $p=2$ zonal jets are almost identical for $F r=0.2$ and $k=$ $-3,-4.5,-6$ (figures not shown). 


\subsection{Physical mechanism of scale selection}

The vertical scale selection has been established in section 6.2.2 using a system of roughly 100 degrees of freedom. However, the relevant physics can be captured by a low-order truncation of the model. An examination of the elements of the matrix $[E]$ in (6.8) corresponding to a given vertical harmonic $p$, reveals that the largest values correspond to interaction terms between the long Kelvin wave at $p$ and the MRG waves at neigbouring $p \pm 1$. This is evidence of a dominance of the lowest meridional mode $r=0$ and interactions between neighbouring vertical modes. For large values of $p$ the meridional profiles of the zonal velocity of the Kelvin wave $D_{0, p}(y)$ and of the meridional velocity of the MRG waves $D_{0, p \pm 1}(y)$ have almost identical shapes. This motivates the analytical determination of the growth rate as a function of $p$ for the restriction of (6.16) to $r=0$ and a single given $p$, thus keeping only the interactions between the basic state horizontal shear flow and a Kelvin wave and a MRG wave, the latter two both of mode $p \dagger$. This yields

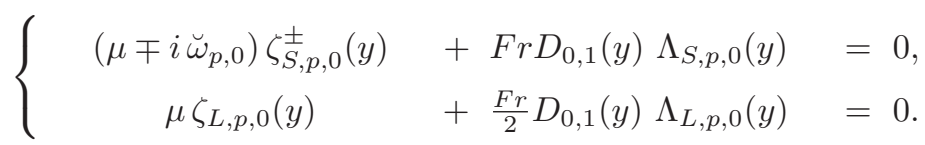

This $3 \times 3$ system is solved by projection onto the meridional dependence of $\zeta_{S, p, 0}^{ \pm}(y)$ and $\zeta_{L, p, 0}(y)$, yielding

$$
\left\{\begin{aligned}
\mu^{2}= & \breve{\omega}_{p, 0}^{2}+2 a_{S} a_{L} \\
a_{S} \equiv & {\left[\frac{\int_{-\infty}^{\infty} \operatorname{Fr} D_{0,1}(y) \Lambda_{S, p, 0}(y) \zeta_{S, p, 0}^{+}(y) d y}{\int_{-\infty}^{\infty}\left(\zeta_{S, p, 0}^{+}(y)\right)^{2} d y}\right] \frac{\hat{v}_{S, p, 0}}{\hat{u}_{L, p, 0}} } \\
a_{L} \equiv & {\left[\frac{\int_{-\infty}^{\infty} \frac{F r}{2} D_{0,1}(y) \Lambda_{L, p, 0}(y) \zeta_{L, p, 0}(y) d y}{\int_{-\infty}^{\infty}\left(\zeta_{L, p, 0}(y)\right)^{2} d y}\right] \frac{\hat{u}_{L, p, 0}}{\hat{v}_{S, p, 0}} . }
\end{aligned}\right.
$$

For large $k, \breve{\omega} \approx-\frac{1}{k}\left(1-\frac{p}{2 k^{2}}\right) \ll 1$, and the growth rate can be approximated as

$$
\mu \approx \operatorname{Frk}\left(\frac{3 p}{4 k^{2}} \frac{\left(2+2 \frac{p}{k^{2}}-3 \frac{p^{2}}{k^{4}}\right)}{1+3 \frac{p}{k^{2}}}\right)^{1 / 2},
$$

confirming that it scales as $\mu \propto F r k$ in agreement with Figure 6(b). Furthermore, $\mu(p)$ has the characteristic bell-shape of scale selection in the larger system, and the maximal growth rate is reached for $p_{\text {peak }} \approx 0.56 k^{2}$, in quantitative agreement with Figures $6(\mathrm{a})$ and $6(\mathrm{~b})$. The growing disturbances are dominated by a long Kelvin wave of meridional characteristic width $\frac{\lambda^{\star}}{\sqrt{p_{p e a k}}} \approx \frac{1}{\sqrt{0.56} k^{\star}}$ and by a MRG wave of comparable meridional characteristic width that has the same zonal wavelength as the basic state. The results are consistent with Figure 4 which shows that the basic state destabilization leads to the formation of high vertical mode zonal jets and zonally-short fluctuations of meridional velocity of comparable vertical scale. Furthermore, Figures $5(\mathrm{c})$ and $5(\mathrm{~d})$ show that the zonal jets can be identified with an eastward propagating Kelvin wave, while meridional velocity fluctuations have westward phase propagation and eastward group velocity, compatible with a high baroclinic mode MRG wave.

The calculation was repeated, neglecting the divergence terms $\left(\delta_{S, p, 0}^{+}+\delta_{S, p, 0}^{-}\right)$of $\Lambda_{L, p, 0}(y)$ in $(6.23)$,

$\dagger$ We have checked that taking $p \pm 1$ and $p$ instead of a single $p$ yield more complicated expressions without changing the results presented below. 
and although the growth rate can change by up to $20 \%$, the value of $p_{\text {peak }}$ only decreases by $4 \%$. We thus infer that the physics of the vertical scale selection lies in the relative vorticity exchanges $V \frac{\partial \zeta^{\prime}}{\partial y}+u^{\prime} \frac{\partial Z}{\partial x}$ between the basic state and disturbances of high baroclinic modes, i.e. in the same terms as are kept in (6.20). We conclude that for the formation of both baroclinic and barotropic zonal jets, the main physical mechanism determining scale selection is barotropic shear instability.

For both baroclinic and barotropic zonal jets, the meridional scale of growing disturbances is close to the zonal scale of the basic state short MRG wave, and a zonal jet disturbance grows simultaneously with a short wave disturbance. The latter has the same zonal scale as and is in phase quadrature with the basic state, allowing it to coherently extract energy.

In closing, let us address the question asked at the beginning of this section: which of barotropic or high baroclinic mode zonal jets will prevail in a given situation? High vertical mode zonal jets require the growth of long Kelvin wave disturbances and as such will develop eastward of a localized zonally short MRG wave, while barotropic zonal jets will develop in domain geometries enabling the (westward) propagation of long nondivergent Rossby waves, for example in the periodic re-entrant channel geometry.

\section{Discussion}

\subsection{Summary}

We have investigated numerically the destabilization of Mixed Rossby Gravity (MRG) waves using threedimensionally consistent, high resolution simulations of the continuously stratified primitive equations and provided a rationale for the vertical scale selection of the zonal jets which appear in the simulations. The numerical exploration has been performed for several types of configurations, namely a zonally-periodic MRG wave in a re-entrant channel, MRG waves forced in a basin, and an initially zonally-localized MRG wave in a very long channel, to distinguish whenever possible the different signals that are excited through their eastward/westward energy propagation.

We have mostly focused on the limit of short westward-propagating MRG waves, where destabilization occurs through barotropic shear instability due to the dominant $\frac{\partial V}{\partial x}$ shear component. The basic state short wave having primarily meridional motions across lines of constant Coriolis parameter $f$, its largescale instability produces zonal jets as shown by Manfroi \& Young (1999). We have found that, overall, the vertical and meridional scales selected in the high resolution stratified primitive equations simulations are consistent with the scales we obtained through a stability analysis of the vorticity equation linearized around a zonally-short MRG wave basic state.

For a re-entrant channel the destabilization leads to barotropic zonal jets dominated by westward flow at the equator and having eastward extra-equatorial flow. On the other hand, for geometries selecting principally eastward propagating signals, the zonally-short MRG wave destabilization leads to very high vertical mode baroclinic zonal jets, resembling the equatorial deep jets observed in situ in all oceans. The main result concerning the vertical scale selection of the equatorial deep jets is that the vertical mode 
number of the dominant mode grows like the square of the dimensional period of the unstable MRG wave.

An interesting question arises from these two different types of response (barotropic versus very highly baroclinic zonal jets). Are these responses related to the concepts of global and local modes of instability introduced by Pierrehumbert (1984) for the destabilization of a zonally varying flow? Global modes of instability, which propagate away from the zone of instability, require cyclic zonal boundary conditions for their existence. The re-entrant channel in which we observe barotropic zonal jet formation has periodic boundary conditions and allows the eastward propagating basic state to continually interact with the westward propagating barotropic signal. Local modes, on the other hand, have a peak downstream of an extremum of the basic state, but grow exponentially within the unstable region. The formation of baroclinic zonal jets which propagate in the same direction as the basic state is more akin to the excitation of modes of this second category.

\subsection{Scale selection for mid-latitude and equatorial $\beta$-plane zonal jets}

Both the analytical model and the numerical simulations have demonstrated that, in the short MRG wave limit, the dimensional vertical mode $p^{\star}$ of the jets that are generated is only determined by the basic state period $T^{\star}$ and is independent of the basic state vertical mode. This distinctive vertical scale selection can be understood when combining properties of mid-latitude barotropic Rossby wave dynamics and stratified equatorial dynamics as follows.

Firstly, Gill (1974) predicts that a short enough Rossby wave of zonal wavenumber $k^{\star}$ will destabilize into zonal jets of meridonal wavenumber $l^{\star}$ such that

$$
l^{\star} \approx 0.6 k^{\star} \text {. }
$$

Since in the short wave limit, MRG waves have the same non-divergent behaviour as mid-latitude barotropic Rossby wave, this scaling must also apply to short MRG wave destabilzation.

Secondly, when transposing this result to the equatorial $\beta$-plane, the key idea is that the meridional scale of the zonal jets is linked to the radius of deformation $\lambda_{p}$ of their vertical mode, which satisfies $\lambda_{p} \propto 1 / \sqrt{p^{\star}}$. From Gill's result this implies

$$
p^{\star} \propto k^{\star 2}
$$

Finally, making use of the property that in the short wave limit the equatorial MRG wave frequency is inversely proportional to the square of their zonal wavenumber and independent of their vertical mode, the vertical scale selection of the jets can be inferred to satisfy

$$
p^{\star} \propto T^{\star 2}
$$

In other words, shorter wavelength, longer period MRG waves destabilize in favour of zonal jets with narrower meridional scale and thus of higher baroclinic mode, and the vertical mode of the zonal jets is proportional to the square of the period of the basic state MRG wave. Such a square law dependance is linked to the equatorial radius of deformation decrease as the square root of the vertical mode number. 
7.3. Comparison of vertical scale selection between Inertial Instability, Parametric Subharmonic Inertial Instability and barotropic shear instability

In the search for an explanation for the oceanic Equatorial Deep Jets (EDJs), Inertial Instability (II) and Parametric Subharmonic Inertial Instability (PSII) have been previously invoked, as they would explain the observed homogenization of angular momentum (Hua et al. 1997; d'Orgeville \& Hua 2005). However, the vertical scale of II has a shear-dependent upper bound and would in any event require an unrealistic shear amplitude to produce vertical structures of the same size as the observed EDJs in the Atlantic ocean (Gouriou et al. 2001). On the other hand, the vertical scale of the barotropic shear instability mechanism reported here has a taller upper bound, and the mechanism provides a rationale for the formation of the observed EDJs from flow with realistic characteristics (d'Orgeville et al. 2007). Moreover, barotropic shear instability induces east-west velocity currents that alternate in direction with depth, whereas neither II nor PSII can create such a flow without some form of damping because their nonlinear evolution is such that the vertical and meridional circulation cells eventually decay, accompanied by stabilisation of the mean flow (Griffiths 2003a). Nevertheless, the imprint of the redistribution cells can be seen in the density field after stabilization, and such imprints have been seen at smaller vertical scales within observed westward equatorial currents in the equatorial Atlantic ocean (d'Orgeville et al. 2004). It is therefore likely that the vertical scale of the EDJs arises from barotropic shear instability while PSII (or II) acts at smaller vertical scales and enhances the homogenization of angular momentum.

\section{REFERENCES}

Baldwin, M. P., Rhines, P. B., Huang, H.-P. \& McIntyre, M. E. 2007 The jet-stream conundrum. Science 315, $467-468$.

Bouchut, F., Le Sommer, J. \& Zeitlin, V. 2005 Transport and mixing during the breaking of balanced and unbalanced equatorial waves. Chaos 15, doi: 10.1063/1.1857171.

Bourles, B., Andrie, C., Gouriou, Y., Eldin, G., du Penhoht, Y., Freudenthal, S., Dewitte, B., Gallois, F., Chuchla, R., Baurand, F., Aman, A. \& Kouadio, G. 2003 The deep currents in the eastern equatorial Atlantic ocean. Geophys. Res. Lett. 30, (5), 8002, doi:10.1029/2002GL015095.

BOYD, J. 1998 Weakly nonlocal solitary waves and beyond-all-orders asymptotics. Kluwer, 610 pp.

Domaracki, A. J. \& Loesch, A. Z. 1977 Nonlinear interactions among equatorial waves. J. Atmos. Sci. 34, $486-498$.

D'Orgeville, M. \& HuA, B. L. 2005 Equatorial inertial-parametric instability of zonally symmetric oscillating shear flows. J. Fluid Mech. 531, 261-291.

D'Orgeville, M., Hua, B. L. \& Sasaki, H. 2007 Equatorial deep jets triggered by a large vertical scale variability within the western boundary layer. J. Mar. Res. 65, 1-25.

D’Orgeville, M., HuA, B. L., Schopp, R. \& Bunge, L. 2004 Extended deep equatorial layering as an imprint of inertial instability. Geoph. Res. Let. 31, L22303.

Dunkerton, T. J. 1981 On the inertial stability of the equatorial middle atmosphere. J. Atmos. Sci. 38, 23542364.

Firing, E. 1987 Deep zonal currents in the central equatorial Pacific. J. Mar. Res. 45, 791-812. 
Frisch, U., Legras, B. \& Villone, B. 1994 Large-scale Kolmogorov flow on the $\beta$-plane and resonant wave interactions. Physica D 94, 36-56.

Fruman, M., HuA, B. L. \& Schopp, R. 2008 Equatorial zonal jet formation through the barotropic instability of a low-frequency mixed Rossby-gravity wave, equilibration by inertial instability, and transition to superrotation. J. Atmos. Sci. (to be submitted).

GILl, A. E. 1974 The stability of planetary waves on an infinite beta-plane. Geophys. Astrophys. Fluid Dynamics 6, 29-47.

Gill, A. E. 1982 Atmosphere-Ocean Dynamics. Academic Press, 662pp.

Gouriou, Y., Andrie, C., Bourlès, B., Freudenthal, S., Arnault, S., Aman, A., Eldin, G., Du Penhoat, Y., Baurand, F., Gallois, F. \& Chuchla, R. 2001 Deep circulation in the equatorial Atlantic ocean. Geophys. Res. Lett. 28, (5), 819-822.

GRIfFIths, S. D. $2003 a$ The nonlinear evolution of zonally symmetric equatorial inertial instability. J. Fluid Mech. 474, 245-273.

Griffiths, S. D. $2003 b$ Nonlinear vertical scale selection in equatorial inertial instability. J. Atmos. Sc. 60, 977-990.

Hua, B. L., Moore, D. W. \& Le Gentil, S. 1997 Inertial nonlinear equilibration of equatorial flows. J. Fluid Mech. 331, 345-371.

IGA, K. 1995 Transition modes of rotating shallow water waves in a channel. J. Fluid Mech. 294, 367-390.

Lee, S. Y. \& Smith, L. 2002 Stability of Rossby waves in the $\beta$-plane approximation. Physica $D$.

MajdA, A. 2003 Introduction to PDEs and Waves for the Atmosphere and Ocean. Courant Institute Lecture Notes in Mathematics,American Mathematical Society.

Majda, A. \& Klein, R. 2003 Systematic multi-scale models for the tropics. J. Atmos. Sci. 60, 393-408.

Manfroi, A. J. \& Young, W. R. 1999 Slow evolution of zonal jets on the beta-plane. J. Atmos. Sci. 56, 784-800.

Manfroi, A. J. \& Young, W. R. 2002 Stability of $\beta$-plane Kolmogorov flow. Physica D 162, 208-232.

Matsuno, T. 1966 Quasi-geostrophic motions in the equatorial area. J. Met. Soc. Japan 44, 25-43.

Meshalkin, L. D. \& SinAi, I. G. 1961 Investigation of the stability of a stationary solution of a system of equations for the plane movement of an incompressible viscous liquid. Appl. Math. Mech. 25, 1140-1143.

Natarov, A., Richards, K. \& J.P. McCreary, J. 2008 Two-dimensional instabilities of time-dependent zonal flows: linear shear. J. Fluid Mech. 599, 29-50.

Reznik, G. \& Zeitlin, V. 2007 Interaction of free Rossby waves with semi-transparent equatorial waveguide. part 1. wave triads. Physica D 226, 55-79.

Rhines, P. B. 1994 Jets. Chaos 4, 313-339.

Richards, K., Maximenko, N., Bryan, F. \& Sasaki, H. 2006 Zonal jets in the Pacific. Geoph. Res. Let. .

RIPA, P. 1983 Weak interactions of equatorial waves in a one-layer model. Part I: general properties. J. Phys. Oceanog. 13, 1208-1215.

Shchepetkin, A. \& MCWilliams, J. 2005 The regional oceanic modeling system: A split-explicit, free-surface, topography-following-coordinate ocean model. Ocean Modelling 9, 891-902.

Sivashinsky, G. 1985 Weak turbulence in periodic flows. Physica D 17, 243-255.

Vallis, G. \& Maltrud, M. 1993 Generation of mean flows and jets on a beta plane and over topography. J. Phys. Ocean. 23, 1346-1362. 


\section{Appendix A. Intermediate lengthscale MRG wave}

In section 3, the case of intermediate lengthscale MRG waves has been mentioned. MRG waves with $k=-1.5$ also undergo an adjustment but on a much longer time scale compared to the short MRG waves studied throughout this paper, and the adjustment does not lead to the disappearance of the inital MRG wave. In order to investigate the nature of the weakly nonlinear interactions, we have performed different initial value simulations keeping the same value of the MRG wave zonal wavenumber $k=-1.5$ and varying the Froude number of the basic state between $F r=0.1$ and $F r=0.4$. In all of the simulations a substantial adjustment occurs with exponentially growing higher baroclinic mode signals, but in all cases the initial MRG wave persists for at least 100 of its periods.

In the first phase of the adjustment, resonant triad (RT) interactions are observed, involving free MRG and Kelvin waves that have substantially higher vertical modes than the initial basic state MRG wave of vertical mode $m^{\star}$. Specifically, for various levels of initial noise in the simulation, the types of RTs that have been observed are such that a Kelvin wave of dimensional vertical mode $p_{K}^{\star}$ and zonal wavenumber $-q k^{\star}$, where $q$ is a positive integer $\dagger$, interacts with the basic state and another MRG wave of vertical mode $p_{K}^{\star} \pm m^{\star}$ and zonal wavenumber $(q+1) k^{\star}$ so that the triad interaction rule on the zonal wavenumbers

$$
k^{\star}=(q+1) k^{\star}-q k^{\star}
$$

and the rule that the sum of the meridional mode indices (Domaracki \& Loesch 1977) of the triad members be an odd integer (in this case -1) are both verified, as is the resonance condition on the frequencies of the MRG waves and the Kelvin wave

$$
\omega\left(m^{\star}, k^{\star}\right)=\omega\left(p_{K}^{\star} \pm m^{\star},(q+1) k^{\star}\right)-q k^{\star} c_{p_{K}^{\star}},
$$

where $\omega(s, l)$ denotes the frequency of a MRG wave of vertical wavenumber $s$ and zonal wavenumber $l$.

The value of $q$ that is preferentially selected has been found to depend on the level of the random noise that is superposed on the initial free MRG wave. For a very low noise level (nondimensional values of velocities less than $10^{-5}$ ), only vertical harmonics of the initial free wave are excited and parity conditions further require that the vertical mode of the Kelvin wave $p_{K}^{\star}$ be an even multiple of $m^{\star}$. This yields a $q=2$ resonance with a Kelvin wave of vertical mode $p_{K}^{\star}=8 m^{\star}$ that grows exponentially and interacts with free MRG waves of vertical modes $p_{K}^{\star}-m^{\star}=7 m^{\star}$ and $p_{K}^{\star}+m^{\star}=9 m^{\star}$ in accordance with the prediction of (A 2) $\ddagger$.

This is confirmed by the time series, for a point located at the equator, of the zonal and meridional velocities of the three largest amplitude vertical modes of the simulation for time $t<2.5$ years (Figure

$\dagger$ in general, $q$ can be a rational number in a channel geometry whose zonal extent is much larger than the basic state MRG wave wavelength, but this is not the case in our simulations, where the zonal extent of the channel is set equal to the MRG wave wavelength.

$\ddagger$ For the same value of $F r=0.2$, other initial-value simulations with higher levels of initial noise (nondimensional velocities of $10^{-2}$ ) have shown the possibility to excite RTs for $q=1$, i.e. a Kelvin wave of zonal wavenumber $+\left|k^{\star}\right|$ and vertical wavenumber $4 m^{\star}$ that interacts with MRG waves of vertical wavenumbers $3 m^{\star}$ and $5 m^{\star}$ (figures not shown), in accordance with (A 2). 
10). An oscillating growing signal corresponding to a vertical mode $p_{K}^{\star}=8 m^{\star}$ in zonal velocity is observed and it may be checked that it corresponds to a free Kelvin wave with zonal wavenumber $+2\left|k^{\star}\right|$. The meridional velocity signal, on the other hand, is consistent with free MRG waves of vertical modes $p_{K}^{\star}-m^{\star}=7 m^{\star}$ and $p_{K}^{\star}+m^{\star}=9 m^{\star}$ and zonal wavenumber $3 k^{\star}$. The RT growth is exponential for time $t<2.5$ years with a growth rate time scale of 75 days (left panels of Figure 10), and we have confirmed numerically that the growth rate is proportional to $\mathrm{Fr}$ by varying the amplitude of the initial free MRG wave.

Once the RT has reached finite amplitude, zonally-symmetric equatorial currents appear, also with finite amplitudes. They are stationary in time and correspond to the excitation of vertical modes $2 m^{\star}$ and $14 m^{\star}$ (top right panel of Figure 10). Their growth rate is smaller than that of the RT for time $t<2$ years and their higher growth rate at later times seems to derive from the decrease in amplitude of modes $m^{\star}, 7 m^{\star}$ and $9 m^{\star}$. The $p_{J}^{\star}=2 m^{\star}$ and $p_{J}^{\star}=14 m^{\star}$ zonally symmetric current structure remains stationary while the basic state MRG wave of mode $m^{\star}$ persists until beyond time $t \geqslant 3$ years.

For all Froude numbers between 0.1 and 0.4 that have been explored, the adjusted state contains (i) a persistent and dominant signal of the initial MRG wave of mode $m^{\star}$ that remains of finite amplitude after both triad resonance and nonlinear saturation have occurred and (ii) a rich vertical mode spectrum with both zonally variable oscillating meridional velocity components and quasi-steady zonally-symmetric currents.

Finally, we have performed numerical simulations with $\mathrm{Fr}=0.2$ in a basin geometry similar to those reported in section 5.2. Instantaneous zonally-symmetric jets could not be found, unless high-pass filtering of vertical modes was applied to the zonal velocity field. Compared to the shorter MRG wave regime, this regime of intermediate wavelength does not appear to be a robust mechanism for producing strongly baroclinic nonlinear zonal jets in a basin geometry. 


\section{Appendix B. Scaling of equation (6.2)}

The goal of this Appendix is to present a scaling of equation (6.2) through a nondimensionalization based on the spatial scales of highly baroclinic disturbances in order to find which are the relevant terms of the linearized vorticity equation. Such an approach thus depends on the properties of high vertical mode disturbance scales rather than on the scales of the MRG wave basic state as was the case for the nondimensionalisation done in section 3.

Taking into account the baroclinic disturbances scaling of section 6.1.1, we can introduce

$$
(U, V, W, \mathcal{Z})=\left(\frac{\omega}{k} \widehat{U}, \widehat{V}, \frac{\omega^{2}}{k} \widehat{W}, k \widehat{\mathcal{Z}}\right), \quad\left(u^{\prime}, v^{\prime}, w^{\prime}, \zeta^{\prime}\right)=\left(\hat{u}^{\prime}, \hat{v}^{\prime}, k^{-1} \hat{w}^{\prime}, k \hat{\zeta}^{\prime}\right),
$$

and redefining space variables using

$$
k x=\xi, \quad k y=\eta, \quad p z=\varsigma,
$$

gives

$$
\begin{array}{r}
k \frac{\partial \hat{\zeta}^{\prime}}{\partial t}+\hat{v}^{\prime}-\frac{p}{k^{2}} \eta \frac{\partial \hat{w}^{\prime}}{\partial \varsigma} \\
+F r\left[\left(\omega \widehat{U} \frac{\partial}{\partial \xi}+k \widehat{V} \frac{\partial}{\partial \eta}+\frac{\omega^{2}}{k} p \widehat{W} \frac{\partial}{\partial \varsigma}\right) k \hat{\zeta}^{\prime}+\left(k^{2} \hat{u}^{\prime} \frac{\widehat{\partial \mathcal{Z}}}{\partial \xi}+\hat{v}^{\prime} \frac{\widehat{\partial \mathcal{Z}}}{\partial \eta}+\hat{w}^{\prime} \frac{\widehat{\partial \mathcal{Z}}}{\partial \varsigma}\right)\right. \\
\left.-\omega^{2} \hat{\zeta}^{\prime} \frac{\widehat{\partial W}}{\partial \varsigma}+k \omega^{2} p \frac{\widehat{\partial W}}{\partial \xi} \frac{\partial \hat{v}^{\prime}}{\partial \varsigma}+\frac{\partial \hat{w}^{\prime}}{\partial \xi} \frac{\widehat{\partial V}}{\partial \varsigma}-\omega^{2} p \frac{\widehat{\partial W}}{\partial \eta} \frac{\partial \hat{u}^{\prime}}{\partial \varsigma}-\frac{\omega}{k} \frac{\partial \hat{w}^{\prime}}{\partial \eta} \frac{\widehat{\partial U}}{\partial \varsigma}\right]=\mathcal{O}\left(F r^{2}\right),
\end{array}
$$

where hat terms are of order $O(1)$ and terms of order $F r^{2}$ have been discarded.

The notations $\frac{\widehat{\partial \dot{ }}}{\partial \xi}, \frac{\widehat{\partial \cdot}}{\partial \eta}, \frac{\widehat{\partial \cdot}}{\partial \varsigma}$ have been used to represent the $O(1)$ parts of the spatial derivatives of the primary MRG wave, which does not vary on the same scales as the disturbances.

Making use of the scaling relations $\omega \sim k^{-1}$ and $p \sim k^{2}$ and keeping the leading terms in $k$ gives

$$
k \frac{\partial \hat{\zeta}^{\prime}}{\partial t}+\hat{v}^{\prime}-\frac{p}{k^{2}} \eta \frac{\partial \hat{w}^{\prime}}{\partial \varsigma}+\operatorname{Fr} k^{2}\left(\widehat{V} \frac{\partial}{\partial \eta} \hat{\zeta}^{\prime}+\hat{u}^{\prime} \frac{\widehat{\partial \mathcal{Z}}}{\partial \xi}-\frac{p}{k^{2}} \widehat{\mathcal{Z}} \frac{\partial \hat{w}^{\prime}}{\partial \varsigma}\right)=0 .
$$

For the regimes we have explored, $\operatorname{Fr} k^{2} \gtrsim 1$, and one can infer the scale of the growth rate of high baroclinic mode disturbances from a balance between the time derivative term $k \frac{\partial \hat{\zeta}^{\prime}}{\partial t}$ and the terms representing the disturbance interactions with the basic state. Since the interaction terms are of $O\left(F r k^{2}\right)$, the growth rate must scale as $F r|k|$.

For non-divergent "barotropic" disturbances, $\frac{\partial \hat{w}^{\prime}}{\partial \varsigma}=0$, and the linearized vorticity equation is

$$
k \frac{\partial \hat{\zeta}^{\prime}}{\partial t}+\hat{v}^{\prime}+\operatorname{Fr} k^{2}\left(\widehat{V} \frac{\partial}{\partial \eta} \hat{\zeta}^{\prime}+\hat{u}^{\prime} \frac{\widehat{\partial \mathcal{Z}}}{\partial \xi}\right)=0
$$

again implying a growth rate of barotropic disturbances which scales as $F r|k|$. 


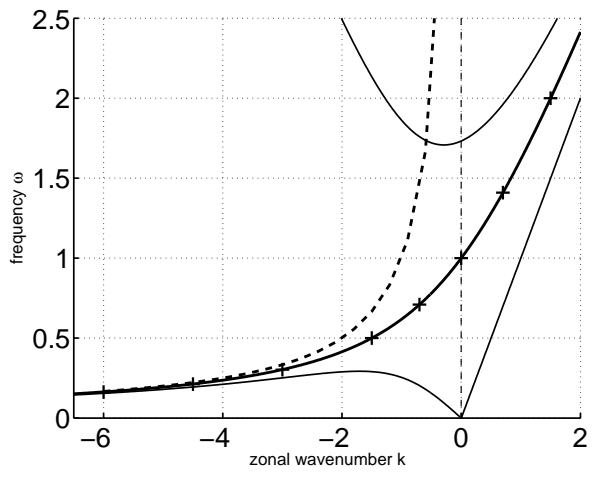

FiguRE 1. Dispersion relation for free equatorial waves of a given vertical mode $m$. Axes have been nondimensionalized by $\sqrt{\beta c_{m}}$ for frequency and by $\sqrt{\frac{\beta}{c_{m}}}$ for zonal wavenumber. Crosses indicate the values of $k$ for which initial-value MRG wave simulations have been performed. The dashed line corresponds to the dispersion relation for zonally propagating mid-latitude barotropic Rossby waves.

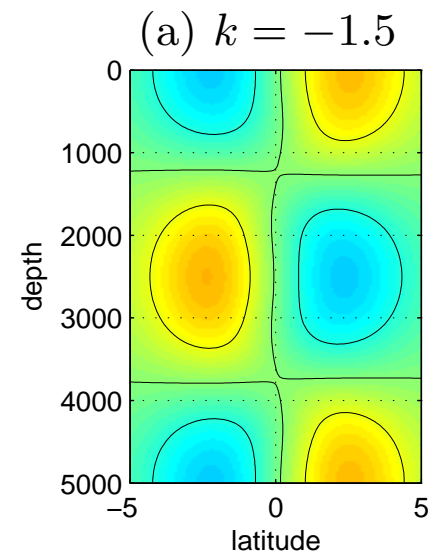

(b) $k=-3$

(c) $k=-4.5$

(d) $k=-6$
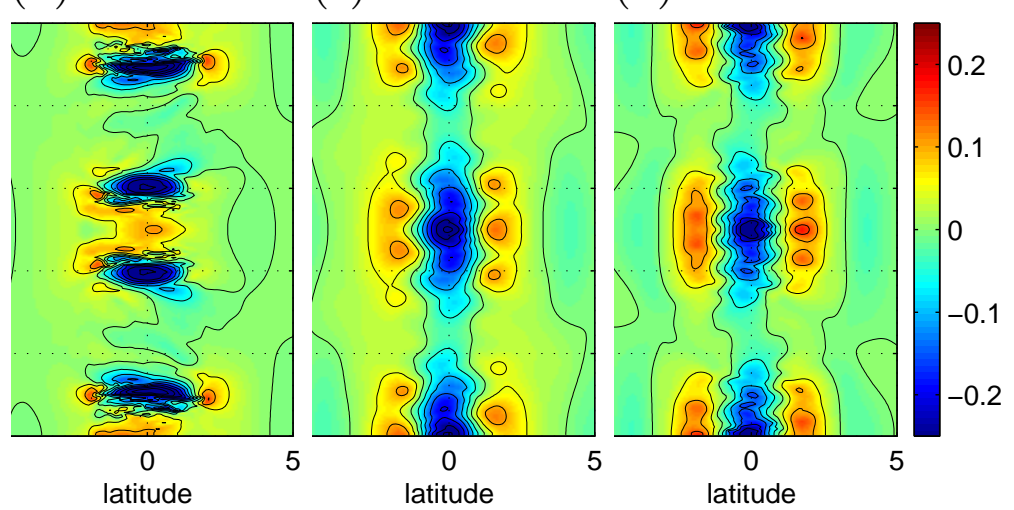

(e) $k=-1.5$

(f) $k=-3$
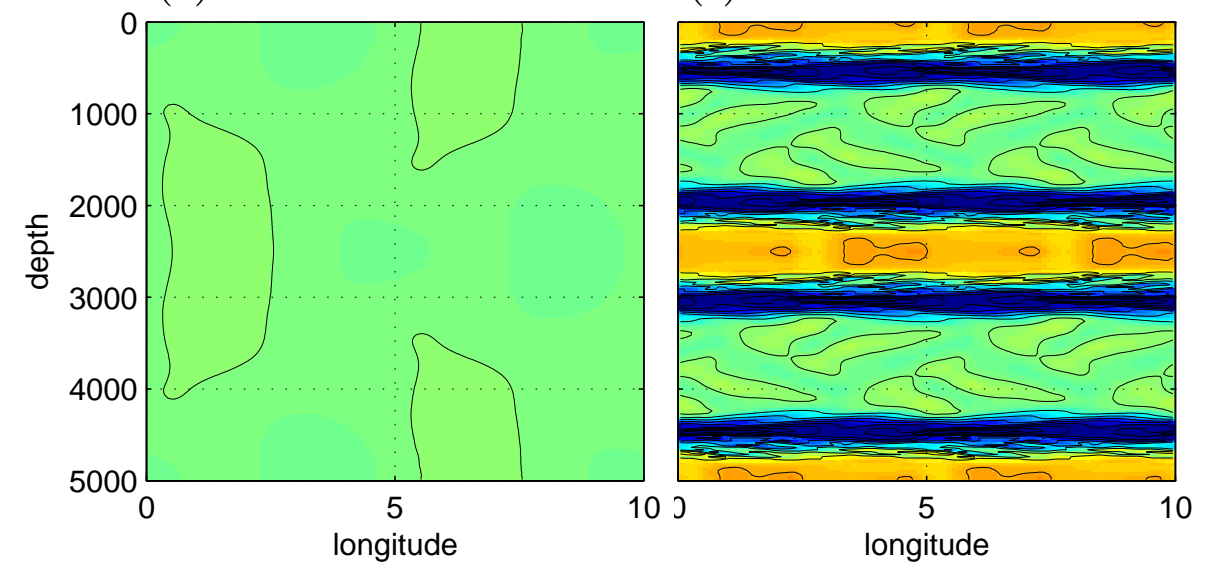

Figure 2. (Top) Depth-latitude instanteneous distribution of zonal velocity for initialized MRG waves of (a) $k=-1.5$, (b) $k=-3$, (c) $k=-4.5$ and (d) $k=-6$. All cases correspond to a vertical mode of the initial MRG wave $m^{\star} H / \pi=2$ and an initial meridional Froude number $\mathrm{Fr}=0.2$. (Bottom) Depth-longitude instantaneous distribution of zonal velocity along the equator for the cases (e) $k=-1.5$ and (f) $k=-3$. 


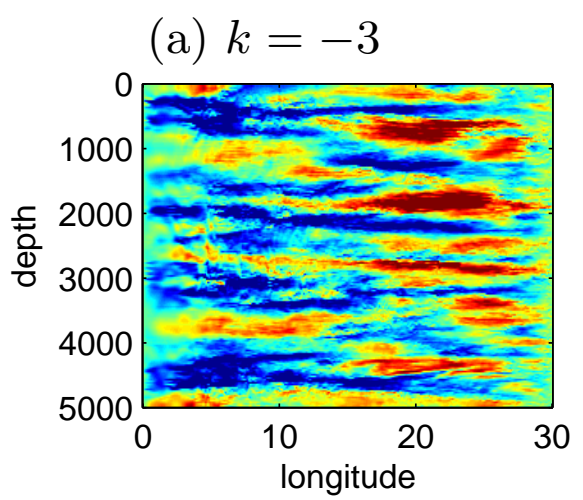

(b) $k=-4.5$

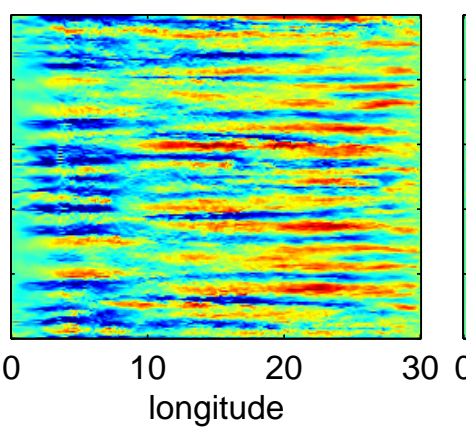

(d) $k=-6$ (c) $k=-6$

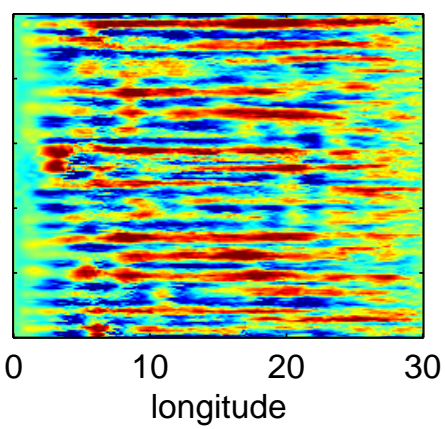

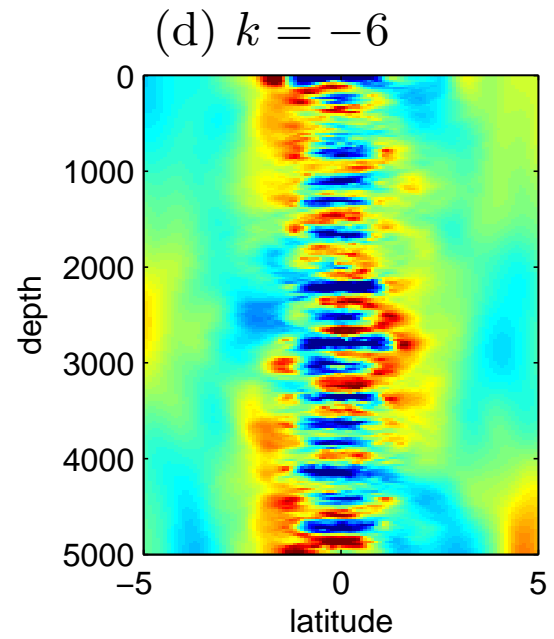

Figure 3. (a)-(c) Equatorial sections of instantaneous zonal velocity for basin simulations with an oscillating forcing in the western part of the basin that excites a free MRG wave of (a) $k=-3$, (b) $k=-4.5$ and (c) $k=-6$. (d) Meridional section of instanteneous zonal velocity at mid-basin for the case $k=-6$. 

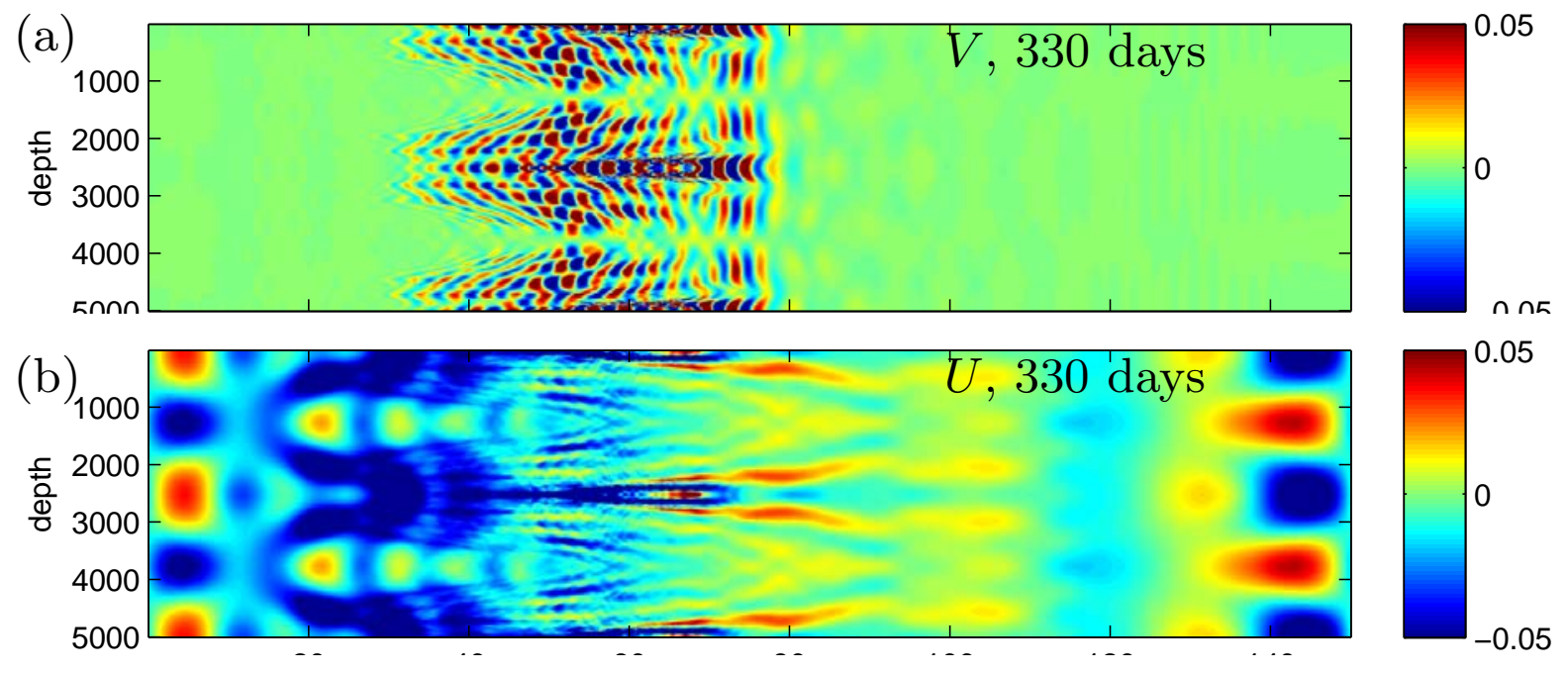

(c)
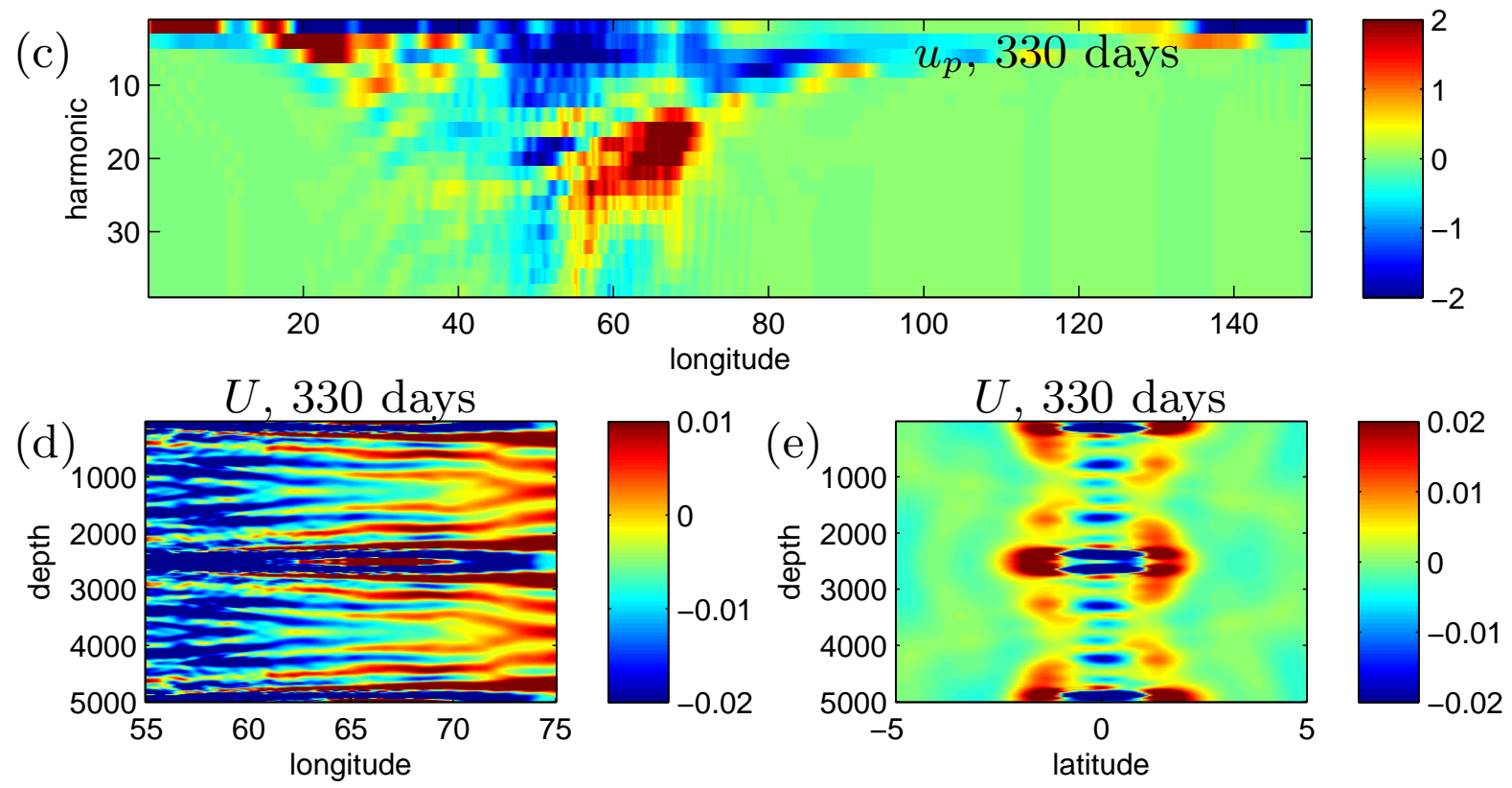

Figure 4. Numerical simulation of a MRG wave with $k=-6$ and $F r=0.2$, initially zonally-localized in the longitude interval $40^{\circ}<x^{\star}<60^{\circ}$. Instantaneous sections along the equator after 330 days of integration of (a) meridional velocity component and (b) zonal velocity component. (c) Vertical mode spectrum of zonal velocity along the equator nondimensionalized by $m^{\star} H / \pi$ (only even harmonics are plotted). (d) Zonal velocity along the equator, close-up on the $55-75^{\circ}$ longitude interval. (e) Meridional section of zonal velocity at $60^{\circ}$ longitude time-averaged over a MRG wave period . 

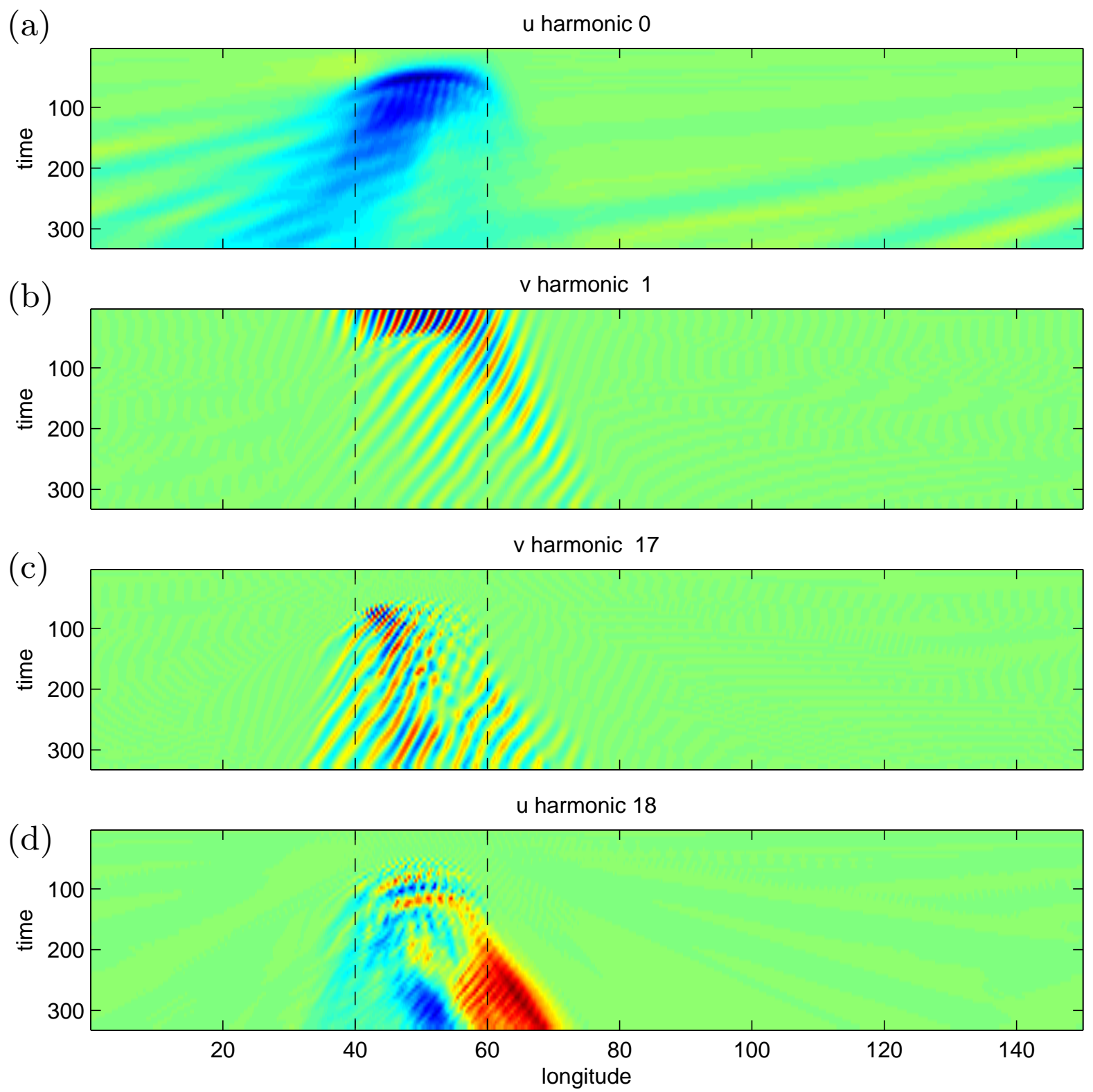

Figure 5. Same simulation as in Figure 4. Hovmöller diagrams for (a) the barotropic component $(p=0)$ of zonal velocity, (b) the meridional velocity of the basic state $(p=1)$, (c) the meridional velocity of harmonic $p=17$ and (d) the zonal velocity of harmonic $p=18$. Time units are in days 

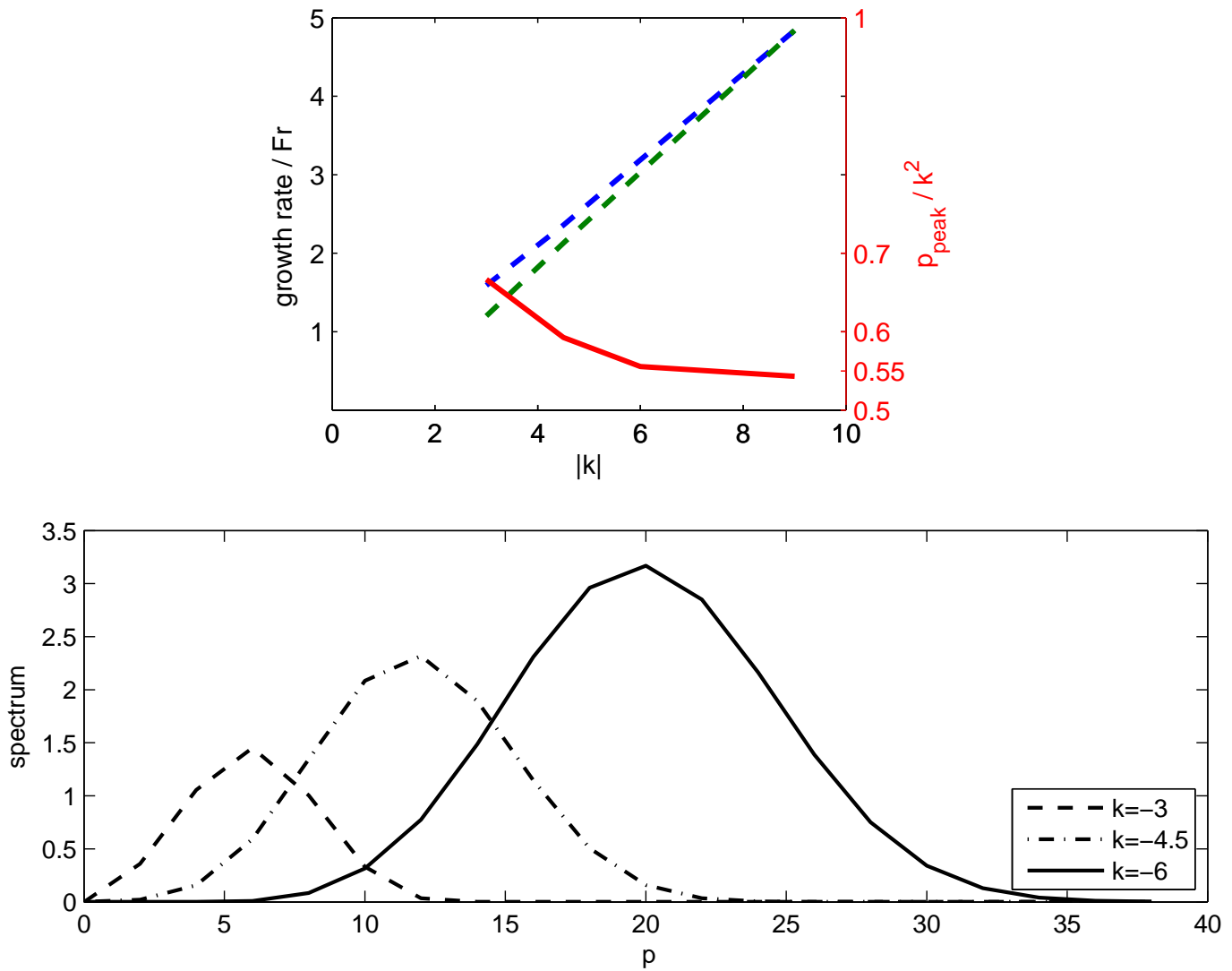

FiguRE 6. (a) Left axis: growth rate $\frac{\mu}{F r}$ for $F r=0.1$ and $F r=0.2$ (upper and lower dashed curves respectively); right axis and red curve: vertical scale selection $\frac{p_{p e a k}}{k^{2}}$. (b) Vertical mode spectra of the zonal jet velocity at the equator for the most unstable disturbance weighted by growth rate for $F r=0.2$ and $k=-3,-4.5,-6$ (red, green and blue, respectively).

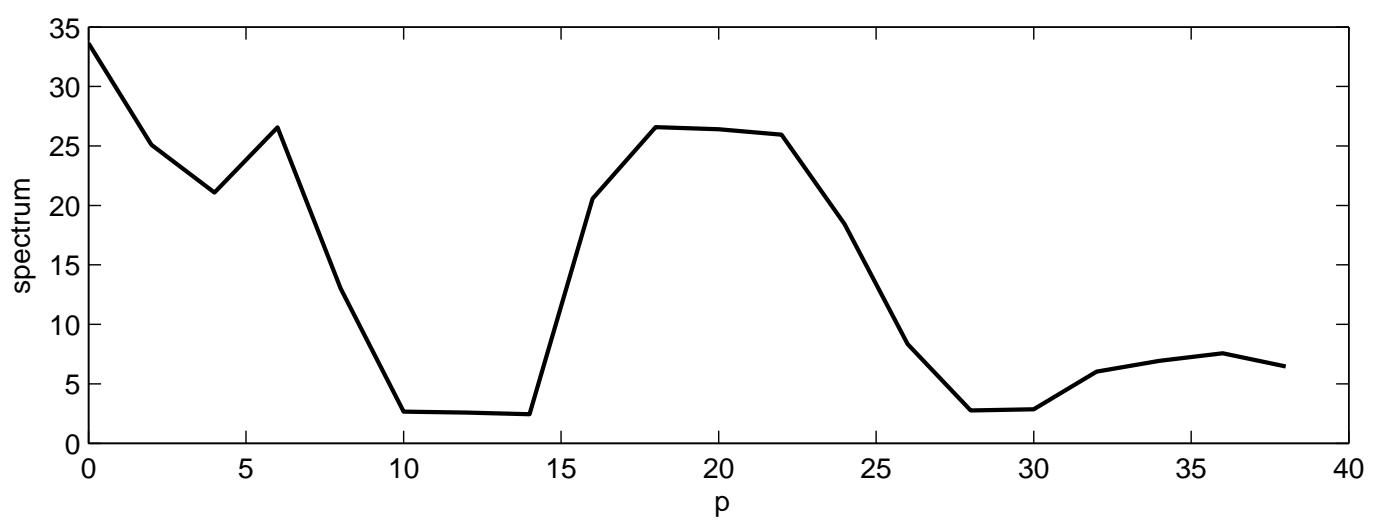

FiguRE 7 . Vertical mode spectrum of zonal velocity at $60^{\circ}$ longitude at the equator in numerical simulations of zonally-localized MRG wave of $k=-6$. 

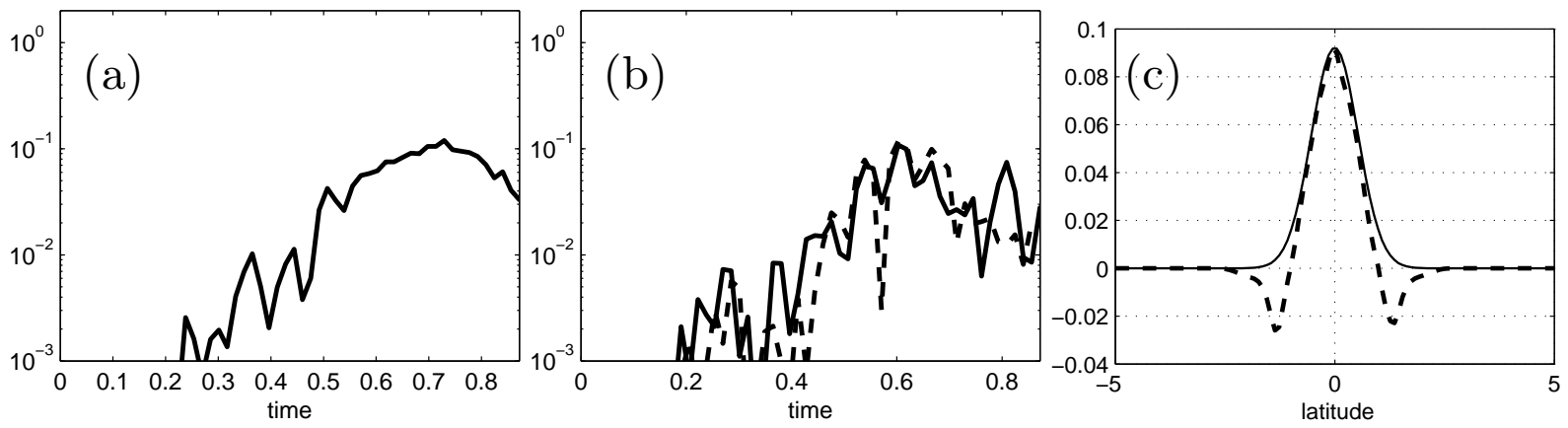

FigURE 8. Results of the simulation for a zonally-localized MRG wave in a long channel geometry at $60^{\circ}$ longitude for $\mathrm{k}=-6$. Time evolution (in years) of (a) zonal velocity harmonic $p=20$ and (b) meridional velocity harmonic $p=19$ (continuous line) and $p=21$ (dashed line)in log-lin coordinates. (c) Meridional structure of the zonal velocity harmonic $p=20$ from numerical simulation (dashed line) and most unstable meridional structure predicted theoretically (continuous line).
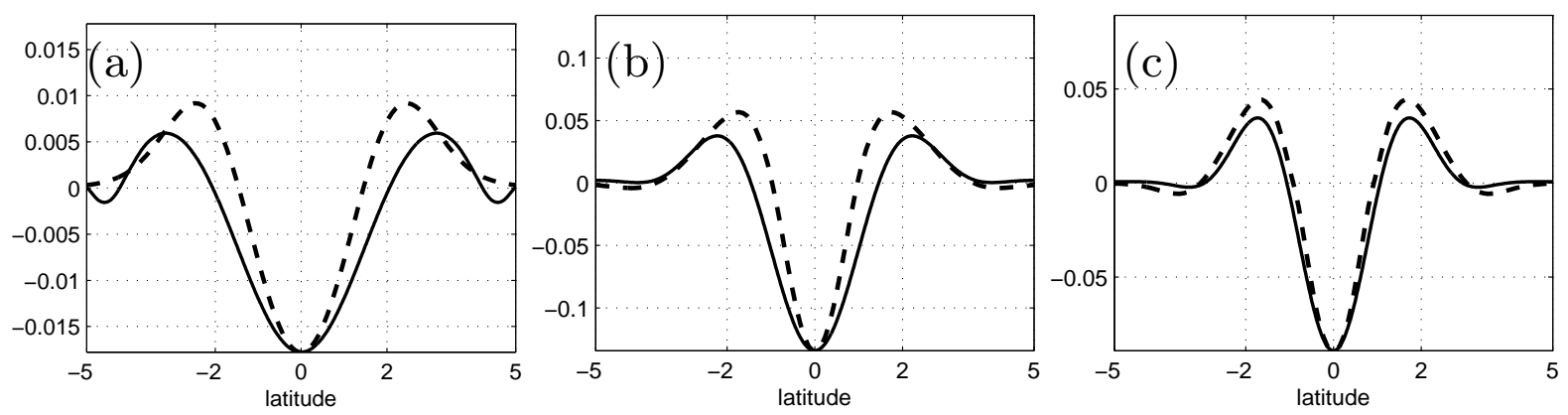

FigURE 9. Early growth phase of barotropic mode: meridional structure of the zonal velocity field of the barotropic mode (dashed line) for the periodic channel simulations shown in Figure 2 for $k=-3,-4.5,-6$, respectively from left to right. The continuous lines correspond to the meridional structure of the zonal velocity profile of the most unstable mode of system (6.22). Latitude is in degrees. 

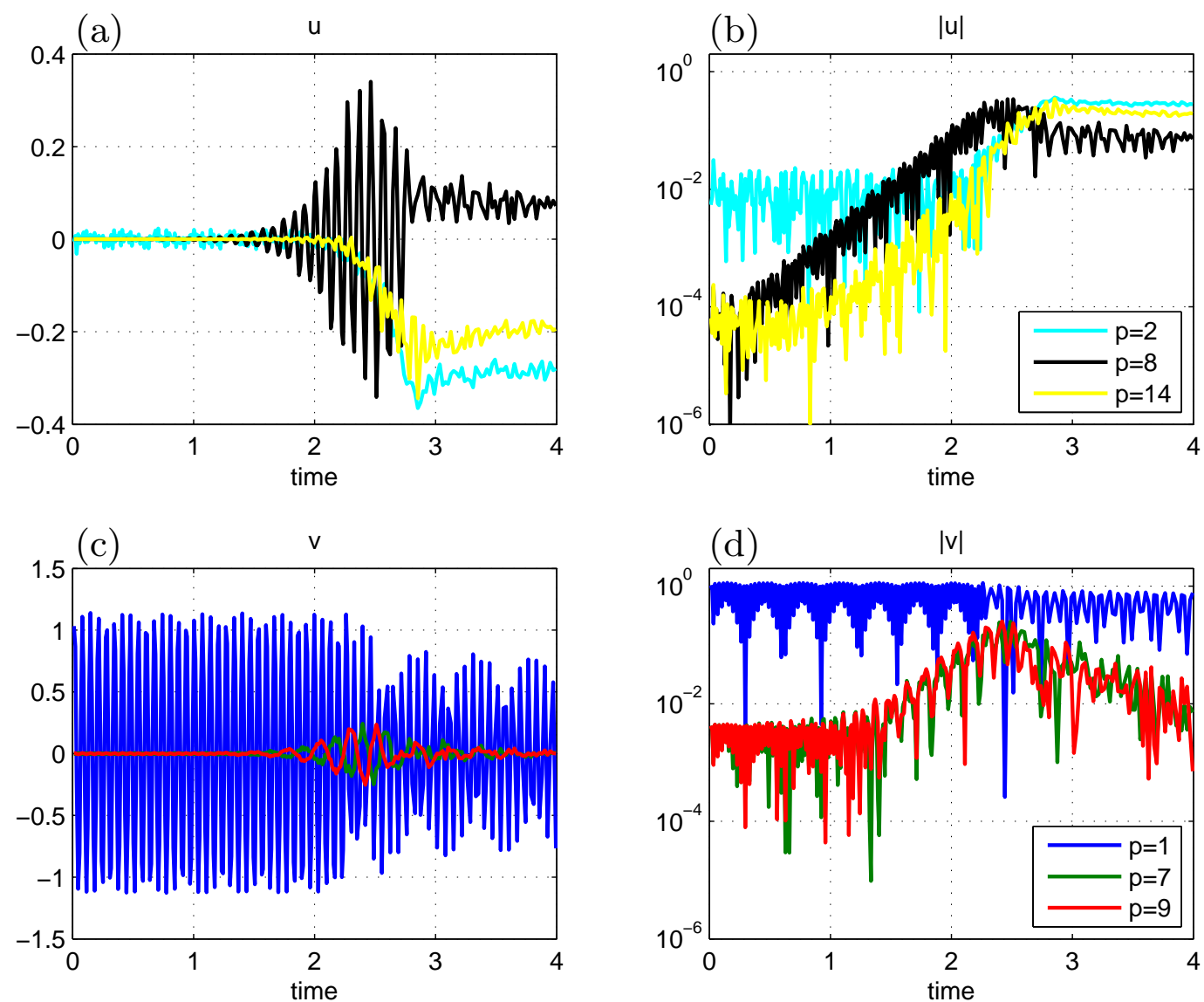

Figure 10. Initial-value simulation for $k=-1.5$ and $F r=0.2$. Time-series (in years) of zonal (top) and meridional (bottom) velocities at a point located at the equator in linear (left) and semilog (right) coordinates for the three vertical modes of largest amplitude. 